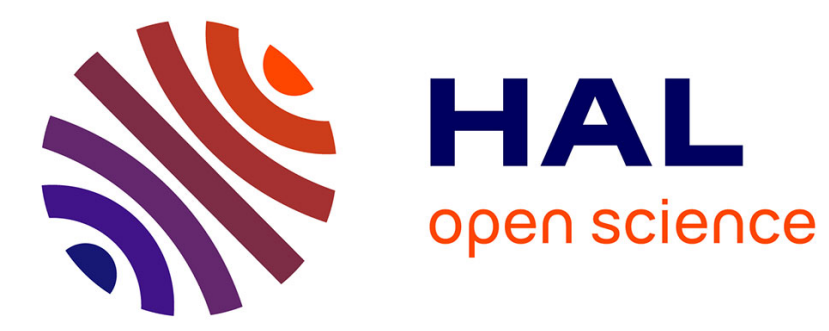

\title{
Les néologismes techniques dans le traité Della cultura degli orti e giardini (1588-1596) de Giovanvettorio Soderini \\ Moreno Campetella
}

\section{- To cite this version: \\ Moreno Campetella. Les néologismes techniques dans le traité Della cultura degli orti e giardini (1588-1596) de Giovanvettorio Soderini. ELAD-SILDA, 2018, Néolex, 1. halshs-02000272}

\section{HAL Id: halshs-02000272 \\ https://shs.hal.science/halshs-02000272}

Submitted on 18 Jun 2020

HAL is a multi-disciplinary open access archive for the deposit and dissemination of scientific research documents, whether they are published or not. The documents may come from teaching and research institutions in France or abroad, or from public or private research centers.
L'archive ouverte pluridisciplinaire HAL, est destinée au dépôt et à la diffusion de documents scientifiques de niveau recherche, publiés ou non, émanant des établissements d'enseignement et de recherche français ou étrangers, des laboratoires publics ou privés. 


\title{
Les néologismes techniques dans le traité Della cultura degli orti e giardini (1588-1596) de Giovanvettorio Soderini
}

\author{
Moreno Campetella \\ Institut Catholique de Lyon, Institut de linguistique romane "Pierre Gardette " / \\ Université Jean Moulin Lyon 3, Centre d'Etudes Linguistiques (CEL - EA 1663), \\ mcampetella@univ-catholyon.fr
}

Résumé : Le traité Della cultura degli orti e giardini du Florentin Giovanvettorio Soderini (1526-1596), dont la première édition, non datée, remonterait aux années 1590, compte parmi les tout premiers textes italiens, sinon le premier, où l'horticulture fait l'objet d'une étude complète et systématique et ses différents aspects abordés de façon scientifique. En témoignent les centaines de néologismes techniques contenus dans cet ouvrage: loin de constituer un élément secondaire dans le développement de cette branche de l'agronomie, l'invention d'un vocabulaire agronomique et horticole moderne représente une preuve irréfutable de la capacité des savants et des professionnels de cette époque à ordonner le monde de la Nature et à maîtriser une certaine technique mieux que leurs prédécesseurs du Moyen âge, souvent encore très dépendants de la science classique, et à s'affranchir des enseignements des Anciens. Parmi les domaines parmi lesquels les phénomènes néologiques sont les plus fréquents rappelons ici la nomenclature des plantes et fleurs, la physiologie végétale ou encore la pédologie ou science des sols.

Mots-clés: Giovanvettorio Soderini, agronomie, horticulture, lexicologie, néologismes, Italie, 16 e siècle

\begin{abstract}
Technical neologisms in Giovanvettorio Soderini's horticultural treatise Della cultura degli orti e giardini, written between 1588 and 1596, demonstrate the highly scientific and linguistic level of Italian agronomists at the end of the Renaissance. It's highly likely that Soderini drew most of the neologisms from the popular country language (i.e. tenerume, meaning the "bud" of a plant), or he created some others to define new natural phenomena (i.e. aovato, meaning an oval leaf). In any case, he is the "inventor" of modern horticultural terminology. Some words show that cultural exchanges between France and Italy were very frequent.
\end{abstract}

Keywords: Giovanvettorio Soderini, agronomy, horticulture, lexicology, neologisms, Italy, $16^{\text {th }}$ century 


\section{Introduction}

\section{L'œuvre et le contexte historique}

La quasi-totalité de la vie de Giovan Vittorio Soderini nous est inconnue. Selon la notice biographique que lui consacre la Biographie universelle de Joseph François et Louis-Gabriel Michaud (T. 42, p. 569), il serait né en 1526 à Florence, au sein de la même famille, appartenant à la petite noblesse de robe, que le bien plus célèbre Pierre Soderini, qui avait instauré une sorte de dictature dans la ville sur l'Arno en accaparant la charge de gonfalonier à vie en 1502. Après des études de droit et de philosophie à Bologne, Soderini aurait participé à un complot contre les Médicis. Condamné par le Conseil des huit à la décapitation il fut gracié par la générosité de Ferdinand $\mathrm{I}^{\mathrm{er}}$ qui le relégua, pour la vie, a Cédri, près de Volterra ${ }^{1}$. C'est là que Soderini «adoucit l'ennui de cet exil en étudiant l'agriculture, en composant des ouvrages estimés sur cette science $^{2} \gg$. Il y mourut le 3 mars 1596. C'est presque exclusivement par son œuvre agronomique, assez conséquente, que son souvenir a été transmis à la postérité. Son traité de viticulture, intitulé Trattato della coltivazione delle viti e del frutto che se ne può cavare, fut publié à Florence par l'éditeur Giunti en 1600, in-4 $4^{\circ}$. Ses autres écrits, portant sur le même domaine technique, ont tous été édités à partir de 1811 à partir des textes originaux manuscrits ${ }^{3}$. Ces derniers ont été transmis sous la forme de quatre volumes autographes conservés dans le fond Magliabecchiano de la Bibliothèque Nationale de Florence, côtés Magl. II.IV.74-77 (ancienne côte Magl. XIV, 42-45).

Le traité Della cultura degli orti, qui fait l'objet de cette contribution, est contenu dans le volume II (Magl. II.IV.75, anciennement ms. Strozzi $n^{\circ} 1177$ ) et consiste en 205 feuilles numérotées au recto ${ }^{4}$. Il aurait été rédigé entre 1588 et la mort de l'auteur ${ }^{5}$. La première édition moderne date de $1814^{6}$.

\footnotetext{
1 Son expérience dans l'art des jardins devait dater de l'époque précédant son exil, s'il est vrai, comme l'indique Giuseppe Sarchiani, éditeur du texte de 1814, sur la base de l'analyse des Libri dell'Ufizio delle decime, que Giovan Vittorio Soderini possédait dans le territoire (contado) à proximité immédiate de Florence, près de Porta alla Croce, une « casa signorile, o Villa con giardino » (p. VII). Cette villa périurbaine était encore la propriété de son fils, Pier Tommaso en 1594. Sur cette propriété voir Lucchesi - Bertocci [1984] ; Lazzaro [1990 : 26].

2 C'est en tout cas ce qui découle de la lecture des premières feuilles des volumes manuscrits.

3 Il s'agit du Trattato d'agricoltura (Firenze, Stamperia del Giglio, 1811, in- $4^{\circ}$ ), Della cultura degli orti e giardini, du Trattato degli arbori (Firenze, Stamperia del Giglio, 1817, in- $4^{\circ}$ )

4 Bacchi Della Lega, 1903 : IX-X.

5 Scriptus post annum 1588, in Villa Cedria, scriptus ubi Tomus I ( $\mathrm{f}^{\circ} 4 \mathrm{r}$ ). Cette indication manuscrite date du début du XIX ${ }^{\mathrm{e}}$ siècle.
}

6 Della cultura degli orti e giardini, Firenze, Stamperia del Giglio, 1814, in-4. C'est cette édition qui, par commodité, a été utilisée pour cette contribution, étant donné qu'aucun écart par rapport à l'édition la plus moderne, celle contenue dans Bacchi Della Lega 1903, n'a été constaté. 
Cet ouvrage, qui a dû compter parmi les textes phares en ce domaine ${ }^{7}$, est bien le signe de l'importance qu'on accorde à l'agronomie, en Italie comme dans le reste de l'Europe, tout le long du Cinquecento. C'est ainsi que ce siècle s'ouvre avec le Libro de agricultura que es de la labrança y criança de Gabriel Alonso de Herrera (Alcalà, 1513), suivi par le De agricultura opusculum d'Antonino Venuti (Naples, 1516), le Traité Von Ursprung der Teilung [...] des Ecker, Wyngarten, Krautgarten de Jacob Koebel, (Oppenheim, 1522) ou encore l'Hortus gallicus de Symphorien Champier (Lyon, 1533). Cet engouement pour les pratiques agraires prend tout son essor à partir du milieu des années 1540 avec des ouvrages tels que La coltivazione de Luigi Alemanni (Paris, 1546), Les propos rustiques de Maistre Leon Ladulphi de Noël Du Faïl (Lyon, 1547). Les traductions des textes de l'Antiquité classique et tardive sont tout aussi incontournables : c'est le cas de la traduction italienne des Libri de re rustica de Columelle par Pietro Lauro (De l'agricoltura, Venise, 1544), de L'opera di agricoltura, version italienne des Ruralium commodorum opus de Pietro De' Crescenzi (Venise, 1553) ou encore, en France, les Ephemerides aeris perpetuae, seu popularis et rustica tempestatum astrologia d'Antoine Mizauld (Paris, 1554). La littérature ayant trait, plus particulièrement, au jardinage se taille évidemment la part du lion à une époque où les espaces verts constituent un moyen privilégié pour véhiculer un message politique ou pour montrer une richesse nouvellement acquise. On peut se limiter à évoquer ici Le plaisant jardin d'Eville de Passebreme (Lyon, 1556) ou les Hortorum libri triginta de Benoît Le Court (Leyde, 1560). Mais ce sont les Italiens les maîtres à penser en la matière, de Bartolomeo Taegio, auteur de La villa (Milan, 1559) et du Hortorum cura (Paris, 1560) à Costanzo Lanzi qui rédige sa Lettera sopra un pino (Milan, 1560), à Giovanni Tatti dont les Della agricoltura libri cinque paraissent à Venise en 1560, jusqu'à Francesco Sansovino, qui est connu pour La villa di Palladio (Venise, 1561), traduction de l'Opus agriculturae de Palladius $^{8}$. Comme on le voit aisément en parcourant cette liste de titres, certainement non exhaustive, la France et l'Italie, sans être évidemment les seules nations en Europe où jardinage et horticulture ont été exercés à un niveau d'excellence, sont les pays où ces derniers ont conquis leurs lettres de noblesse et qui ont su conserver cette primauté au moins jusqu'au début du XVIII ${ }^{\mathrm{e}}$ siècle. C'est dans ces deux régions européennes que, pour employer les paroles d'Ubrizsy-Savoia [2000b : 379] ces publications témoignent le mieux « de l'attrait qu'exerce encore une agriculture de type aristocratique, qui permet au propriétaire de jouir des délices de la campagne en prenant part à quelques nobles activités telles que le jardinage, si cher aux Anciens et en particulier à Columelle ». Cette volonté accrue de goûter aux plaisirs de la campagne, qui devient une véritable mode à partir des années 1570 , se ressent d'un côté

\footnotetext{
7 L'édition du Trattato della coltivazione delle viti, parue chez Giunti en 1600 est accompagnée du Della coltivazione delle viti e di alcuni arbori de Bernardo Davanzati, rédigé vers 1581. On ne peut pas exclure qu'il s'agisse d'une stratégie commerciale mise en place par le célèbre éditeur florentin pour doper les ventes de l'œuvre de Soderini fraîchement terminée ; mais il est tout aussi vraisemblable que la science de ce dernier en matière d'horticulture était déjà assez réputée pour qu'un texte sorti vingt ans plus tôt puisse bénéficier de la publicité de sa summa sur l'aménagement du potager et du jardin. Sur la notoriété de Soderini et sur son importance comme source pour l'histoire des jardins, voir Lazzaro [1990: 5].

8 Ubrizsy-Savoia [2000:454-455]; Ubrizsy-Savoia [2000b : 375-379].
} 
comme de l'autre des Alpes; c'est tout de même dans la Péninsule, qui, en cette fin de Cinquecento, continue de jouer pleinement son rôle de repère culturel en ce qui concerne l'aménagement aussi bien des jardins que des potagers - culture des fleurs et culture des légumes du potager ne faisant qu'un depuis le début du Moyen âge ${ }^{9}$ - qu'elle prend les formes les plus ostentatoires. Des textes comme celui auquel Agostino Gallo consacre toute sa vie, Le vinti giornate dell'agricoltura et de' piaceri della villa (Venise, 1572), en élargissant au fil des années les originaires Dieci giornate, éditées en 1550, en est un parfait exemple, mais aussi, plus proche de Soderini, Il vago e dilettevole giardino (Pavie, 1593) de Giovanni Rinaldi ou Le Giardino di Agricoltura ... ove si tratta di tutto quello che s'appartiene à sapere a un perfetto giardiniere (Venise, 1592) ${ }^{10}$. C'est dans ce background culturel qu'il faut replacer le Trattato degli orti e giardini de Giovanvettorio Soderini. Son ouvrage est bien le signe d'une époque où le travail des champs, dont ces écrits dressent le portrait, n'évoque aucunement le dur labeur du paysan luttant pour éviter la famine ${ }^{11}$ mais bien plutôt la gaité et l'insouciance (otium) qu'avait dû éprouver Cicéron prenant ses aises dans sa villa de Tusculum ou Pline Le Jeune dans celle de Laurentes. Malgré les nombreux conseils pratiques prodigués par ces intellectuels - et Soderini ne fait pas exception à la règle - dans ce type de production scientifique la vie à la campagne relève beaucoup plus du jeu littéraire que de l'existence réelle. Elle est presque exclusivement synonyme de villas et des domaines, souvent très étendus, qui les entouraient. Soderini ne parlait, de ce point de vue précis, que d'un phénomène qu'il connaissait directement s'il est vrai, comme rappelé plus haut, qu'il possédait deux vastes domaines, le premier près de la Porta alla Croce dans la proche banlieue de Florence, le deuxième à Cedri, dans le territoire de Volterra.

\section{La langue du Della cultura degli orti e giardini}

Cette veine bucolique, assez proche du monde de l'idylle théocritéen, qui parcourt les textes agronomiques de la seconde moitié du $\mathrm{XVI}^{\mathrm{e}}$ siècle, explique en grande partie les choix linguistiques de leurs auteurs. En effet, les plus célèbres d'entre eux sont rédigés en langue vernaculaire, qu'il s'agisse de la traduction d'une œuvre de l'Antiquité ou d'un traité original, comme dans le cas du Trattato degli orti. Soderini opte donc pour l'italien, ce qui constitue l'aboutissement d'un processus entamé depuis les années 1540 au moins : celui-ci voit le vulgaire triompher de façon de plus en plus nette sur le latin, en particulier dans les disciplines

\footnotetext{
9 Toussaint-Samat [1987: 447-449].
}

10 Au sujet de cette mode " champêtre » au sein de l'aristocratie ou de la haute bourgeoisie italienne de la seconde moitié du XVI" siècle, voir Saltini [1984 : 285-293]. A partir des années 1540 les vigne (vignobles) étaient devenues un élément incontournable pour tous les maîtres d'œuvre à qui on confiait le dessin d'une villa extra-urbaine. Sur la Nature et la campagne artificielles mises en place dans les villas de la Renaissance italienne voir Lazzaro [1990 : 8-19, 109-130].

11 Pourtant la période qui s'ouvre au début des années 1540 est bien celle qui a connu quelques-uns des hivers les plus rudes de l'époque qui a précédé le "petit âge glaciaire » du XIX ${ }^{\mathrm{e}}$ siècle. Ces catastrophes climatiques durent durement toucher toutes les récoltes, même dans le Sud de l'Europe (Le Roy Ladurie [1983 : 12-13]) 
techniques. Parmi ces dernières, celles destinées à la divulgation ${ }^{12}$ - cela semble être le cas du traité Degli orti - adoptent le toscan comme langue officielle et uniquement admise ${ }^{13}$. L'Opus de Soderini marque également l'apogée d'une nouvelle tendance éditoriale qui se manifeste en Italie à partir des années 1550-1560, celle des publications en langue vulgaire : dans les maisons vénitiennes Giolito De' Ferrari et Marcolini, deux des plus importantes de la Péninsule, les œuvres en italien s'élèvent à $95 \%$ du total dans la seconde moitié du siècle, chez les Giunti de Florence, le premier éditeur du Degli orti, jusqu'à $75 \%{ }^{14}$. Cette stratégie de la part des principales maisons de la Péninsule n'était évidemment pas absente au commencement du siècle mais au milieu du Cinquecento la production en vulgaire est sans commune mesure supérieure, d'un point de vue quantitatif, à celle des cinquante années précédentes. Le choix de l'italien au détriment de la langue de Cicéron est très vraisemblablement dicté par la montée en puissance d'une nouvelle classe sociale, la bourgeoisie marchande : cette dernière accapare des tranches de plus en plus importantes de pouvoir économique et politique et affiche sa réussite par l'achat de terres. En montrant les mêmes signes extérieurs de richesse que les aristocrates, ces parvenus essaient d'atteindre le même statut social que ces derniers et d'obtenir une reconnaissance qui, souvent, leur fait encore défaut. Or, ces nouveaux propriétaires fonciers ne maîtrisent pas le latin qui était l'idiome officiel des sciences, dont l'agronomie. Il fallait donc que celui qui s'attelait à la tâche de les guider dans leur recherche des joies de la vie champêtre puisse s'adresser à ces nouveaux lecteurs de la façon la plus compréhensible possible ${ }^{15}$. Le traité d'agriculture de Soderini est, de ce point de vue, particulièrement représentatif de cette attitude, inédite auparavant, de l'auteur vis-à-vis des destinataires de son œuvre.

Les innombrables phénomènes néologiques du Degli orti e giardini, dont les exemples qui suivent ne représentent qu'une infime partie, sont la marque non pas d'un agronome de profession qui serait en train de créer de toutes pièces un technolecte adapté à un domaine inconnu. On peut légitimement supposer que la plus grande partie des lexèmes ${ }^{16}$ dont il sera question dans les pages qui suivent devaient faire partie depuis des siècles de la langue, très populaire, des habitants des campagnes toscanes (basilecte), qui les employaient pour identifier objets et phénomènes de leur quotidien. Cet aspect du technolecte «sodérinien »

12 Formentin [1994 : 182, 204-207]; Dionisotti [1967b : 242-243].

13 Parmi celles-ci les volgarizzamenti de textes de l'Antiquité constituent des pièces maîtresses. A ce sujet Dionisotti [1967 : 125-168]; Marazzini [1993 : 29-41] ; Formentin [1994 : 182, 204-207] ; Frosini [2014]. En Toscane, la langue de Dante commence à concurrencer sérieusement celle de Cicéron, même dans le Studium de Florence, dès le début du siècle même si la première chaire de lettore di favella toscana est créée seulement en 1588 par Ferdinand $\mathrm{I}^{\mathrm{er}}$.

14 Trovato [1994: 149-160]; Formentin [1994: 182].

15 Exemplaires, de ce point de vue, sont les traductions italiennes des œuvres en latin de Charles Estienne (1504-1564), le Seminarium seu plantarium et le De re hortensi libellus (1536), par Pietro Lauro (Seminario et Delle herbe, 1545). Sur l'élargissement du public des lecteurs dans l'Italie du XVI e siècle voir Grendler [1993 : 453-454] ; Grendler [1995] ; Richardson [1994 : 90-108] ; Librandi [2003] ; ThébaudSorger [2016]. Sur la victoire définitive de l'italien sur le latin à partir du milieu du XVI siècle voir Trifone [1993 : 431-437]; Serianni [1993 : 496-504]; Simon [2016].

16 Le terme « lexème » constitue dans cet article un synonyme de « mot ». 
constitue l'axe de recherche le plus important dans cette publication, deux tiers au moins des termes examinés en représentant des exemples assez probants. L'importance de Soderini ne consiste donc pas en une créativité lexicale particulière mais en une attitude toute naturelle à enregistrer des vocables qui, dans le cas contraire, ne seraient pas arrivés jusqu'à nous : qu'il décrive la physiologie végétale des plantes du potager ou la granulométrie du sol, domaines extrêmement techniques s'il en est, nombreux sont les termes qui paraissent avoir été puisés au langage des rustres et qui font pendant à d'autres mots d'origine savante ${ }^{17}$. Cela est encore plus vrai de la plupart des nomenclatures vernaculaires : c'est par le biais de son œuvre que plusieurs variétés anciennes de légumes, désormais disparues, nous sont connues ${ }^{18}$. A travers cet agronome amateur, c'est tout un monde rural qui reprend vie sous nos yeux. C'est la raison principale qui est derrière la répartition des mots analysés dans cet article, thématique plutôt que linguistique au sens propre, plus apte donc à mettre en lumière la faculté de l'auteur à photographier les différentes manifestations de la société de l'époque et à les conserver pour la postérité.

C'est également grâce à cette précieuse banque de données lexicales qu'est le Degli orti que nous sommes renseignés sur les échanges de savoirs intervenus entre jardiniers français et italiens. Si la complexité et la maniabilité du technolecte est bien le miroir du haut niveau scientifique d'une civilisation et que langue spécialisée et épistème sont bien les deux facettes d'un même système ${ }^{19}$, alors la terminologie des sciences agraires «sodérinienne » est bien à l'aune de l'avance des spécialistes de la Péninsule sur leurs confrères transalpins : cela découle de l'examen d'un certain nombre de néologismes ayant trait en particulier à l'étude des sols ou à l'aménagement des parcs et jardins. Le fait que la plupart des néologismes du Degli orti se soient perpétués dans la littérature agronomique des siècles suivants, jusqu'à nos jours, témoigne on ne peut mieux de la modernité de cette langue de spécialité de la fin de la Renaissance : cette modernité lui est conférée par un haut degrée de précision, parfaitement en phase avec l'un des paramètres fondamentaux du langage scientifique contemporain qui est celui de la biunivocité du rapport signifiant-signifié (un seul nom pour chaque chose, une seule chose pour chaque nom $)^{20}$.

L'idiome de Soderini se fonde donc sur ce noyau dur qu'est le patrimoine lexical paysan auquel notre théoricien puise et qu'il officialise en quelque sorte; cela en constitue la

\footnotetext{
17 Le poids de l'héritage des grands théoriciens italiens de l'art des jardins devait tout de même être fort s'il est vrai que Soderini se serait inspiré du traité d'architecture de Leon Battista Alberti (achevé en 1452) pour la conception des carrés de plantations dont il est question dans le Trattato degli orti (Lazzaro [1990:2]); il y aurait également emprunté une bonne partie de sa terminologie spécialisée (Lazzaro [1990: 45]).

18 Dans un tiers des cas de figure analysés le passage du Trattato degli orti constitue la seule attestation d'un certain technicisme, qui devait pourtant être très répandu à la campagne, ce qui inciterait à nous interroger sur le concept même de hapax.

19 Gille [1964: 188-189].

20 DL s.v. nomenclature. Pour le concept de précision et biunivocité dans les technolectes italiens voir Mammino [1995]. Pour Simon [2016: 259] la langue vernaculaire est véritablement la seule à même d'exprimer les concepts scientifiques et de décrire les phénomènes naturels avec la plus grande concision et précision.
} 
composante principale, sur laquelle viennent se greffer des éléments provenant des langues techniques mises au point par d'illustres prédécesseurs aux $\mathrm{XIV}^{\mathrm{e}}$ et $\mathrm{XV}^{\mathrm{e}}$ siècles. C'est le premier phénomène, tout à fait majoritaire, qui caractérise son œuvre et qui est en même temps la marque du haut niveau atteint par l'agronomie italienne du Cinquecento et des échanges culturels intervenus entre la Péninsule et la France pendant la Renaissance. C'est à tous ces aspects marquants de la langue du Degli orti, en particulier à l'étude de cette récupération $\mathrm{du}$ langage $\mathrm{du}$ bas-peuple, ainsi qu'à son intégration dans la moderne terminologie horticole, indice de son extrême précision et fonctionnalité, qu'est consacrée l'analyse lexicale des néologismes qui suit.

\section{Terminologie botanique}

Plusieurs néologismes techniques du Degli orti concernent la terminologie employée pour décrire différents phénomènes de la physiologie végétale. Ce fait n'est pas surprenant quand on considère que le $\mathrm{XVI}^{\mathrm{e}}$ siècle est l'époque où la science botanique prend son essor. Ce développement s'annonce depuis le début du siècle avec les premières traductions latines des textes botaniques de Téophraste, telles que De historia plantarum... de causis, sive generatione plantarum... Theodoro de Gaza interprete (Bâle, 1534), sans oublier les ouvrages vernaculaires de vulgarisation comme par exemple, en italien, Dell'historia delle piante, di Theophrasto libri tre, tradutti nuovamente in lingua italiana da Michel Angelo Biondo medico (Venise, 1549). Le renouveau de cette science gagne toute l'Europe dans la première moitié du Cinquecento: ainsi, si quelques œuvres montrent encore un attachement farouche à la tradition ancienne - c'est le cas en France du Grant Herbier, adaptation française du Circa instans, œuvre de l'Ecole de Salerne, qui, après une première édition en 1488, est à nouveau imprimé à Paris en 1521 sous le titre Le Grant Herbier en François, contenant les Qualitez, Vertus et proprietez des herbes, Arbres, Gommes et Semences - le Suisse Anton Schneeberger publie, à Cracovie, un Catalogus stirpium quarundam Latine et Polonice conscriptus (1556), dans lequel des espèces nouvelles, non prises en compte par les Anciens parce qu'endémiques de régions non méditerranéennes, font leur apparition, ce qui oblige les savants à une description des plus détaillées, conséquence directe de l'observation directe du monde de la Nature. L'illustration botanique est également un signe évident de cette renaissance scientifique : après les premières illustrations de Leonard de Vinci et Albrecht Dürer entre 1520 et 1530 , cette nouvelle tendance trouve ses meilleures manifestations dans les œuvres d'Otto Brunfels (1488-1534), Hieronymus Bock (1498-1554) et Leonhart Fuchs (1502-1566). En ce qui concerne plus spécifiquement l'histoire des langues, de l'italien en particulier, il est aujourd'hui évident que l'évolution et le perfectionnement de la terminologie des sciences naturelles a été souvent une conséquence directe de cet essor de l'illustration scientifique ${ }^{21}$.

21 Mandressi [2016]. 
Tout aussi importantes furent les conquêtes en matière de nomenclature, du Catalogus plantarum latine, graece, germanice et gallice de Conrad Gessner (Zürich, 1532), ou encore le Botanicon (1540) de Théodore Dorsten (1492-1552), jusqu'aux Discorsi sulla materia medica di Pedacio Dioscoride Anazarbeo (1554) du médecin siennois Pietro Andrea Mattioli $^{22}$. L'œuvre encyclopédique de ce dernier n'aurait pas était possible sans la création des premiers jardins botaniques qui voient le jour justement en Toscane au milieu du Cinquecento : le premier, celui de Pise, fut créé en 1543 par Luca Ghini par la volonté de Cosme de Médicis, qui y nomma en qualité de premier conservateur Leonhart Fuchs en personne. Suivit, en 1545, celui de Florence, l'année même de la fondation du plus célèbre d'entre tous, le jardin botanique de Padoue ${ }^{23}$. La seconde moitié du XVI ${ }^{\mathrm{e}}$ siècle constitue l'accomplissement des recherches lancées dans la première. En ce qui concerne l'Italie, il suffit de penser aux différents catalogues dont furent dotés les jardins botaniques, tels que L'horto de i Semplici di Padova (Venise, 1591) de Cortuso, qui dresse une liste de 1168 espèces végétales ${ }^{24}$; mais aussi aux nombreuses études d'histoire naturelle comme le traité Dell'Historia Naturale de Ferrante Imperato (Naples, 1599) contenant un important herbier, ou les herbarii de Gaspard Ratzemberger (constitué à Heidelberg entre 1556 et 1592) et de Jacob Horst, sans doute le plus célèbre de tous, entamé dès 1587 mais publié à Marbourg seulement en 1630. Rien de plus normal, dans ce contexte, que les néologismes du Degli orti soient particulièrement abondants dans le domaine précis de la description du monde de la Nature. Les innovations lexicales et sémantiques forgées par Soderini, loin de constituer de simples étiquettes apposées sur des phénomènes inconnus, représentent au contraire une nouvelle manière d'apprivoiser l'univers qui l'entoure: cette faculté de nommer les différentes composantes du règne végétal est probablement l'héritière du savoir-faire mis en place par ses illustres prédécesseurs depuis le début du siècle.

Parmi ces néologismes qui affinent la terminologie botanique on peut citer les adjectifs (a)ovato (una foglia), synonyme d'ovale (néologisme sémantique) et arborescente (néologisme lexical), caractérisant une plante pérenne à port très érigé et dont la tige aérienne est plutôt épaisse et porte peu de ramifications («arborescent»). Les deux attributs sont employés au sujet de la joubarbe, le premier pour les feuilles d'une espèce de petite taille, le deuxième en tant que déterminant ${ }^{25}$ de la dénomination binomiale identifiant la joubarbe arborescente :

L'aizòon, detto il Semprevivo, è di tre spezie. Il maggiore ha le foglie più lunghe, il minore l'ha aovate. L'uno e l'altro nasce, il minore fra le macie delle muraglie, tra $\mathrm{i}$ sassi, nelle corone delle mura, e nei sepolcri, ove non batte il sole, e su per i tetti, e si

\footnotetext{
22 Au sujet de la « renaissance » botanique de la première moitié du XVI e siècle voir Ubrizsy-Savoia [2000 : 438-450].

23 Sur les jardins botaniques italiens de la Renaissance voir Attlee [2006 : 41-49]; Ubrizsy-Savoia [2000 : 443-446].

24 Ubrizsy-Savoia [2000b : 340-343].

25 Dans cet article, nous utilisons le terme « déterminant » pour désigner toute partie de la phrase accompagnant le nom qui en précise la nature. Il est, dans la quasi-totalité des cas, un synonyme d'« adjectif qualificatif ».
} 
nutrisce ancora nei vasi trapiantato con la pianta in terra. Il terzo è l'arborescente che sta attaccato in terra e cresce in alto, massimamente sostentato da qualche telaio di legname, e va su rampicando ; serve per ispallierette ; e questo a ramo, o spiccato dal corpo vecchio, di primavera s'attacca. Gli altri due con un poco di lor pane di terra ai piedi, ed ancora con la radice...

(Il y a trois sortes d'aizòon, qu'on appelle aussi Semprevivo [joubarbe]. La plus grande en taille possède des feuilles plus longues, la plus petite les a ovales. Toutes les deux, en particulier la forme la plus petite, se développent dans les fissures des murs, parmi les rochers, au sommet des murs d'enceinte des villes, là où on trouve les créneaux, ou encore dans les caveaux des cimetières, là où le soleil ne tape jamais ou sur les toits des maisons. Elles peuvent également être mises en terre, dans un pot. La troisième espèce est arborescente, se développe dans la terre et pousse vers le haut, surtout quand elle peut prendre appui sur un tuteur en bois, sur lequel elle grimpe ; on l'utilise pour en faire des bordures [filets] ; cette dernière espèce, on la multiplie par marcottage, les deux autres on les transplante avec une petite motte de terre attachée aux racines, en faisant attention à ne pas couper ces dernières ${ }^{26}$.) (p. 27)

Arborescente peut également indiquer l'attitude qu'ont les jasmins à s'élever en grimpant à des supports :

I gelsomini del fior giallo hanno più dell'arborescente degli altri, avendo il gambo più grosso e più gagliardo.

(Les jasmins à fleurs jaunes ressemblent plus que les autres variétés aux plantes arborescentes, étant donné qu'ils ont la tige plus grosse et robuste.) (p. 136)

Le premier sémantème attesté de l'adjectif (a)ovato, précédant chronologiquement le témoignage de Soderini, fait référence au domaine de l'architecture, désignant un élément de décoration de forme ovale ${ }^{27}$ (Pier Francesco Giambullari (1499-1555), GDLI I p. 534). Le nom masculin ovato est employé, quant à lui, depuis la fin du $\mathrm{XV}^{\mathrm{e}}$ pour décrire n'importe quelle surface au contour ovale (GDLI XII p. 296 n. 5).

L'épithète qu'on retrouve chez Soderini en représente une spécialisation botanique indiquant une feuille «che ha forma allungata, con la parte basale più larga rispetto alla punta ${ }^{28}$ " (foglia) (GDLI XII p. 296 n. 3). Cette signification technique est largement répandue dans les écrits scientifiques des siècles suivants. Ainsi chez Raimondo Montecuccoli (1609-1680) les

\footnotetext{
26 Toutes les traductions vers le français de cet article sont de l'auteur. Pour des raisons liées à la quantité considérable de sources citées, seuls ont été traduits les passages dont la complexité aurait pu poser des problèmes de compréhension ainsi que tous ceux qui paraissent dans le texte.

27 Pour ce qui est du substantif ovato, une occurrence de ce sens primaire se retrouve dans l'oeuvre, à peu près contemporain du Trattato degli orti, de Giovan Paolo Lomazzo (GDLI XII p. 296 n. 7), où il désigne une fenêtre elliptique ou un ove.

28 Cristoforo Landino emploie déjà ovale dans un contexte botanique mais seulement pour caractériser le fruit du sorbier, non pas en faisant référence à des feuilles : «Le sorbe sono di quattro forme : alcune sono tonde come mele, alcune sono aguzze come père ; alcune hanno forma ovale » (Historia Naturale di C. Plinio secondo di latino in volgare tradotta, Venise, 1534, p. 316. $1^{\text {ère }}$ édition : 1476).
} 
feuilles du tabac sont qualifiées d'ovate ${ }^{29}$. Le botaniste florentin Ottaviano Targioni-Tozzetti donne une définition précise de l'adjectif ovato : "Quanto alla figura della lamina delle foglie, dicesi ovata, quando è più lunga che larga e ambedue le estremità sono molto rotonde $^{30} »$. Il est à remarquer que, dans le domaine de la botanique, l'adjectif ovato n'aurait pas le même sens qu'ovale, "che ha un contorno ellittico con estremità simmetriche " (Enciclopedia Treccani s.v.). Cette distinction est confirmée par une glose du botaniste

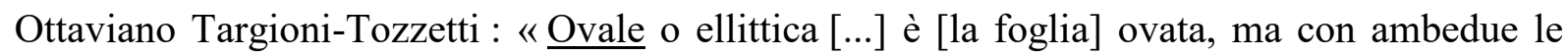
estremità più strette ed eguali ${ }^{31} »$. Le français ové pourrait avoir constitué un emprunt à l'italien botanique (1798, VENTENAT, Tabl. du règne végétal, p. 425, cité dans TLFi s.v.).

Quant à arborescente, il s'agit d'un dérivé du lat. arborescens, participe présent de arborescere (PLINE, Nat. Hist., 19, 62), ce qui symbolise bien l'importance de la science des Anciens dans les savoirs de la Renaissance. Preuve en est le fait que ce déterminant est le seul encore utilisé dans la nomenclature vernaculaire botanique moderne ${ }^{32}$, alors qu'arboreo $\left(<\right.$ lat. arboreus $^{33}$ ) est employé dans les seules dénominations binomiales linnéennes.

L'adjectif occhiuto (hapax sémantique) semblerait désigner, quant à lui, le verticille de la fleur, c'est-à-dire l'« ensemble des organes d'une fleur (étamines, pétales, sépales) insérés circulairement autour de sa partie centrale. » (TLFi s.v.) :

In cima del suo pedale [dell'Oscimoide - Lychnis calcedonica] [...] fa il suo fiore, il quale è incrociato di cinque di larghezza con la sua boccia sotto che gli tiene in sesto come ai garofani fa la sua le sue foglioline.

(Au sommet de la tige [...] se trouve la fleur, qui se compose de cinq éléments [membres] et dont la forme fait penser à un œil en son centre. Elle est d'une remarquable beauté $[\ldots]$ et les éléments qui composent cette sorte de croix à cinq bras ont une longueur et une largeur qui correspondent parfaitement à la taille du calice ; c'est aussi le cas des folioles et du calice des œillets.) (p. 200)

Il correspond, dans la terminologie botanique moderne à «di forma verticillata » («verticillé »). Plus en général verticille peut indiquer l'«ensemble d'organes similaires

\footnotetext{
29 «Le foglie del tabacco sono lunghe, ovate» («Les feuilles du tabac sont longues, ovales », Gimorri [1924: 405]). Chez Giorgio Santi (Viaggi per la Toscana, 1795, p. 146) ovato désigne, non pas les feuilles d'un végétal mais des sortes d'écailles dont le botaniste amateur croit voir revêtu le tronc du monotropa hypopithys, plante parasite du châtaignier : «Il fusto non ha le foglie ma, in quella vece, squame ovato-deltoidee succiliate » («Le tronc ne possède pas de feuilles mais, à leur place des écailles ovales, en forme de delta et dotées, dans sa partie supérieure, d'une sorte de cils »).
}

30 Istituzioni botaniche, vol. I (Florence, 1813), p. 78.

31 «La feuille qu'on qualifie d'ovata peut afficher une forme ovale ou elliptique, mais, par rapport à ces dernières, les deux extrémités sont plus étroites [ = pointues] et symétriques » (Istituzioni botaniche, Florence, 1802, vol. I p. 78). Dans les écrits d'autres auteurs modernes l'acception de l'adjectif pourrait ne pas être celle, technique indiquée par Targioni-Tozzetti. Voir par exemple Luigi Bartolini [1964 : 97] : «Ovali foglie d'acacia ». Sur ovato désignant le profil particulier d'une feuille, voir Stearn [1966: 475] et Lindley [1830: 109]. La distinction entre ovale et ovato n'est plus observée dans la moderne terminologie botanique italienne.

32 Cf. fr. arborescent, attesté pour la première fois chez Pierre Belon (1553 - « Le tithymale arborescent », cité dans TLFi s.v. arborescent). 33 Voir Stearn [1966:387]. 
(feuilles, fleurs, fruits, ramifications secondaires, etc.) insérés circulairement autour d'un axe commun»(TLFi ibid.). La seule acception attestée (depuis le $\mathrm{XV}^{\mathrm{e}}$ siècle) de l'adjectif occhiuto est celle de «constellato di chiazze naturali tondeggianti ${ }^{34}$ ». Le témoignage de Soderini constitue un hapax sémantique. L'absence totale d'autres attestations, ainsi que la forme même de ce déterminant de la physiologie végétale laisserait supposer qu'il s'agit d'une création propre à l'horticulteur de Florence. Il n'en demeure pas moins que les phytonymes populaires, dérivés d'une disposition pour ainsi dire sphérique des pétales ou des autres éléments constituant la structure de la corolle d'une fleur, rappelant la forme d'un œil, sont assez répandus dans la nomenclature vernaculaire, aussi bien italienne que française : œeil de vache (Arnica montana, 1544 - FEW VII, 315b), occhio di gatto (Guilandina Bonduc chez Ottaviano Targioni-Tozzetti ${ }^{35}$ ), occhio della Madonna (Myosotis), occhio di pavone (Trigridia pavonina ou Dianthus arenarius chez Ottaviano Targioni-Tozzetti ${ }^{36}$ ). Ces noms populaires doivent être considérés comme des spécifications techniques qui ont transformé un nom commun originaire (occhio) en autant de noms propres qu'il y avait d'espèces florales qui rappelaient aux locuteurs cette partie du corps. De ce sémantème nous ne possédons aucune attestation écrite mais il a dû circuler oralement pendant des siècles dans les campagnes comme on peut en juger par certaines œuvres littéraires modernes : ainsi, Arturo Graf (1848-1913) s'adresse-t-il de la sorte dans l'un de ses poèmes à une fleur des champs :

\section{O tenero fiorellino / ....su pei margini ignudi / e lungo i rivoli schiudi / il picciol} occhio turchino ${ }^{37}$.

Quelques lignes inspirées à Curzio Malaparte par la contemplation d'un champ de tournesols sont également assez curieuses dans la mesure où l'écrivain décrit ici la même plante analysée par Soderini :

Era proprio una selva di girasoli, una vera foresta : curvi sugli altri steli pelosi, il grande e rotondo occhio nero, dalle lunghe ciglia gialle, annebbiato dal sonno, $\mathrm{i}$ girasoli dormivano a capo chino ${ }^{38}$.

\footnotetext{
34 «Parsemé de taches naturelles de forme vaguement ronde» (GDLIXI p. 777 n. 3). Ce dernier semble avoir été également le seul sémantème attesté dans un contexte botanique en français : oeillé, oeilleté, ocellé, «parsemé de tâches rondes, ayant l'aspect d'un œil » (par ex. la roue du paon), depuis 1230 (FEW VII, p. 313a).

35 « [...] occhiuto in mezzo, di notabilissima bellezza [...] ed i pezzi della croce ch'egli fa in quinto sono equidistanti di lunghezza » (« [...] au centre [de la fleur] il y a un élément circulaire, d'une beauté remarquable [...] et les cinque membres qui l'entourent, semblables au cinq bras d'une sorte de croix ont tous la même longueur », Istituzioni botaniche, Florence, 1813, II p. 63).

36 Pour d'autres témoignages, non datés ou datant du début du XIX ${ }^{\mathrm{e}}$ siècle, voir GDLI XI, p. 768-769 et Beccaria [1995 : 276-278].

37 «Ô toi, ma petite fleur jolie, qui ouvre sur les flancs rocheux des montagnes, ou le long des ruisseaux ton petit œil turquoise » (Le poesie, Turin, 1922, p. 1014, cité dans GDLI XI p. 766 n. 7). Cf. Antonio Fogazzaro : « Il popolo delle passiflore, dei gelsomini, delle altre piante arrampicate a' muri, guardava dall'alto, con mille occhi » (« Le peuple des fleurs de la passion, des jasmins et des autres plantes nous fixait du regard, du haut des murs auxquels il grimpait, avec leurs innombrables yeux », Malombra, 1881, cité dans GDLI, ibid.); Giovanni Papini : «Stringeva nel piccolo roseo pugno una margherita dall'occhio di zolfo » (« Il serrait dans sa petite main rose fermée une marguerite à l'œil couleur du soufre », Il tragico quotidiano, 1906, cit. dans GDLI, ibid.).
} 
Un autre technicisme désignant une partie de la plante à la forme bien particulière est pannocchiuto (néologisme lexical). Il indique une «inflorescence de certaines graminées qui se présente sous la forme d'une grappe d'épillets dont les axes secondaires plus ou moins ramifiés décroissent de la base au sommet de l'axe central » (TLFi s.v.) :

\section{[L'apocino] fa certi fiori pannocchiuti, belli a vedere ma all'odore cattivi. \\ ([La cynanque droite] fait des fleurs paniculées, très belles mais à l'odeur désagréable.) (p. 34)}

Pannocchiuto, qui correspond à panicolato («paniculé ») dans la terminologie actuelle, est dérivé de pannocchia, "infiorescenza composita, disposta a grappoli su assi secondari descrescenti dal basso verso l'alto, di aspetto conico » (depuis les Discorsi su Dioscoride de Pietro Andrea Mattioli, Venise, 1565 - GDLI XII p. 494). Mais le terme panicola, ayant le même sens, est attesté (hapax) dans le Statuto della gabella di Siena (XIII' siècle). Il sera repris par Castor Durante (1529-1590) dans l'Herbario novo (Venise, 1602) pour caractériser les fleurs de l'orme de montagne (GDLI XII p.481). Le Dizionario italiano Tramater (Naples, 1840 s.v.) connaît la forme masculine panicolo (GDLIXII p. 482). L'adjectif employé par Soderini sera largement répandu dans la littérature botanique des décennies suivantes pour désigner toute sorte d'inflorescence paniculée.

Emmanuele Tesauro caractérise de cette façon les fleurs de l'amarante ${ }^{39}$. De la même espèce végétale il est question également chez Bartolomeo Clarici, chez qui le déterminant technique employé est celui en usage chez les modernes botanistes : «Delle molte spezie [di amaranto] che n'abbiamo e che in oggidì sono presso a quaranta, solo quindici ne' giardini se ne coltivano, cioè il cristallo d'un bellissimo rosso, il purpureo, l'altro purpureo, l'oscuramento rosso, l'argenteo, il coccineo massimo pannicolato, il cremise $^{40} »$. Ce dernier passage représente également la toute première attestation italienne de panicolato.

En français les témoignages écrits de termes techniques composés sur la même base lexicale sont chronologiquement antérieurs. Ainsi en est-il de l'adjectif paniculé, -ée, « qui possède des fleurs en panicule ». La première attestation daterait de 1545 (Guillaume Guéroult, Hist.des plantes, cité par TLFi s.v. panicule). Cela ferait pencher pour un caractère paneuropéen du terme, suite vraisemblablement à des échanges de savoirs entre la France et l'Italie, depuis le début du XVI ${ }^{\mathrm{e}}$ siècle.

38 «C'était une vraie forêt de tournesols : ils étaient endormis, les têtes bien repliées, les longues tiges velues recourbées vers le bas. Leurs grands yeux, noirs et sphériques, entourées de longs cils jaunes, étaient sur le point de se refermer, alourdis par le sommeil » (La pelle, 1949, cité dans GDLI, ibid.).

39 « Il pannocchiuto e immortale amaranto » (Panegirici, vol. I, p. 83, cité dans GDLI XII, p. 494)

40 « Parmi les nombreuses espèces [d'amarante] qu'on trouve de nos jours, plus ou moins quarante, il n'y en a qu'une quinzaine qu'on peut cultiver dans les jardins : il s'agit de celui qu'on appelle cristallo, d'une splendide teinte rouge, le pourpre, une autre variété qui tire également sur le pourpre, une autre d'un rouge assez foncé, une autre encore d'une couleur rappelant l'argent, une espèce d'une taille remarquable aux fleurs vermeilles paniculées, le cramoisi » (Istoria e coltura delle piante che son pe 'l fiore più ragguardevoli e più distinte per ornare un giardino, Venise, 1726, p. 734, cité par GDLI XII p. 481). 
D'autres néologismes de cette catégorie thématique définissent non pas la forme des éléments de la plante mais les parties elles-mêmes. Différemment des adjectifs relatifs à la physiologie végétale qu'on vient de passer en revue, ces nouveaux termes semblent appartenir à la langue populaire, au sein de laquelle ils devaient circuler depuis les temps les plus anciens. Un exemple en est le nom de la vrille de la courge ou d'une quelconque cucurbitacée, mandata (néologisme lexical) :

\footnotetext{
Il frutto di alcune è in terra e d'altre fuore, e d'alcuna nell'uno e nell'altro luogo.

Alcune si aggiacciono in terra come il cocomero e la zucca, ed il popone e i cetriuoli, e l'angurie; ma le zucche in pergola fanno maggiori e grandissime mandate sopra gli albori, altre per innalzarsi han bisogno d'appoggio come il convolvolo, la balsamina, i piselli, e dei fagiuoli alcune sorte...

(Le fruit de certaines plantes se trouve sous terre, chez certaines autres il pousse hors terre, d'autres encore aussi bien en dessous et au-dessus de la surface. Certaines espèces, comme par exemple les pastèques de différentes sortes, les courges, les melons, les concombres, rampent par terre; les courges, qu'on installe normalement sur les pergolas, émettent des vrilles bien plus grosses et longues que les premières, en grimpant aux arbres, d'autres encore ont besoin de tuteurs pour pousser, comme par exemple le convolvulus, la balsamine, les petits pois, certaines espèces de haricots.) (p. 14)
}

Il s'agit de toute évidence d'un synonyme de gettata, désignant le « rejet » d'une plante, en particulier de la vigne : cette acception n'est attestée que depuis la première moitié du XVIII ${ }^{\mathrm{e}}$ siècle (Cosimo Trinci, GDLI VI p. 722 n. 3, s.v. gettata) mais le verbe gettare, "pousser (les végétaux, les arbustes)»est connu depuis les XIV (Dante Alighieri, GDLI p. 715 n. 10). Le néologisme mandata est vraisemblablement dérivé du verbe mandare qui, dans le sens de «faire pousser» (des végétaux)», transitif ${ }^{41}$, est attesté dans les plus anciens textes agronomiques italiens (traduction italienne de l'œuvre de Pietro De' Crescenzi, XIV ${ }^{\mathrm{e}}$ siècle) et qui est très employé jusqu'à l'époque moderne (début du XIX ${ }^{\mathrm{e}}$ siècle, GDLI VI p. 621 n. 14). On peut également supposer que le choix d'un synonyme de gettata s'imposait en raison de la nature exotique de la courge en question, probablement une citrouille, récemment introduite à partir du Nouveau Monde ${ }^{42}$. Le terme habituellement employé en italien standard est aujourd'hui ricciolo, littéralement «boucle », en référence a sa forme.

Le substantif nero ${ }^{43}$ (néologisme sémantique) signifie l'occhio, l'ilo (« œil », « hile ») du haricot. Après le témoignage de Soderini et un silence d'un siècle ce sémantème réapparaît à la fin du XVII ${ }^{\mathrm{e}}$ siècle chez Emanuele Tesauro (1592-1675) [1669: 85] : "Ilo o nero della fava » (cit. par GDLI XI p. 373 n. 33). Le dictionnaire trilingue d'Annibale Antonini (1762, T.

\footnotetext{
41 L'emploi intransitif du verbe (« bourgeonner ») est connu seulement par le biais d'un passage du Corso d'agricoltura (vol. IV, p. 48, 1801-1803) de Marco Lastri : «Il sesamo [...] è una pianta erbacea, che manda un caule diviso in più steli » (« Le sésame [...] est une plante herbacée dont la tige est divisée en plusieurs rameux [ = dont la tige, l'inflorescence est composée]».

42 Neveu [1975: 203].

43 Pour le passage de référence voir infra § 2.7.
} 
II p. 770, s.v. viso) en atteste encore l'usage à la fin du XVIII ${ }^{\mathrm{e}}$ siècle : «Viso. Diciamo a quel nero del fagiolo. Petite tache noire qu'on voit sur quelque sorte d'aricot. Un viso rinacagnato di fagiolo ».

Tenerume (néologisme sémantique) est, quant à lui, pour employer la terminologie contemporaine, le rampollo, la gemma, ou encore la rispuntatura («bourgeon ») :

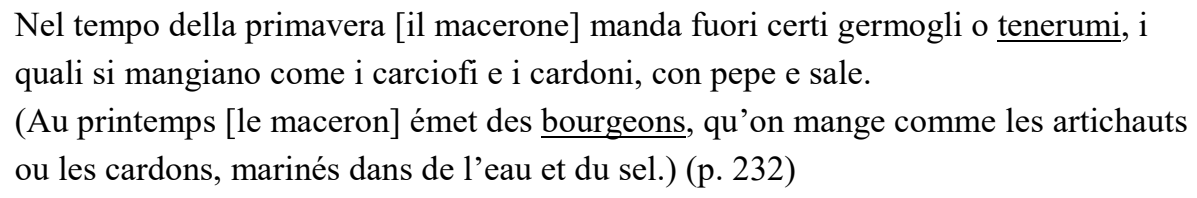

Ce sémantème représente vraisemblablement une spécialisation technique à partir du sens primaire de tenerume, "parte tenera di un vegetale », attesté depuis le XIV siècle (Cresc. Vulg., GDLI XIX p. 887, n. 1). Des mots apparentés sont très répandus au niveau régional dans les aires linguistiques italo-romane et gallo-romane: frioul. tenarùm, a.fr. tendrum, « junger Spross » (REW s.v. "tener ») ; «tenron, bourgeon, rejeton tendre d'arbres" $\left(\mathrm{XIV}^{\mathrm{e}} \mathrm{s}\right.$. $)$, tendron, «bourgeon» (1538, FEW XIII 208a); tendrillon, «bourgeon tendre » (1558, Huguet VII p. 211). Cette répartition géographique laisserait supposer que le lexème en question, encore bien vivant en Toscane (GDLI XX p. 886), appartenait probablement au basilecte agricole.

\section{Nomenclature botanique vernaculaire}

On trouve également dans le Degli orti de multiples néologismes désignant certains légumes et herbes du potager. Ces termes, d'autant plus intéressants qu'ils identifient très vraisemblablement des variétés de végétaux aujourd'hui disparues, devaient circuler oralement au sein du basilecte agricole toscan. D'autres noms - c'est le cas du tournesol, appelé Erba del sole ou de l'amarante (Passavelluto) - nous donnent de précieux repères chronologiques sur l'introduction de plantes et fleurs exotiques en Europe occidentale et sur l'usage qu'on en faisait. Ce fait n'est pas étonnant au vu de l'engouement suscité par cette mode dans les jardins de la fin du $\mathrm{XVI}^{\mathrm{e}}$ siècle $^{44}$. Nous en dressons ci-dessous une petite liste qui est loin d'être exhaustive : 


\subsection{Adraena $=$ Portulaca oleracea L. (porcella comune, porcellana) (« pourpier maraîcher, porcelane »)) (néologisme lexical)}

L'Adraena di campo ha le foglie simili al semprevivo, un poco più rotonde, più piccole e men lunghe e va per terra [...] È la medesima Porcellana.

(L'Adraena des champs a les feuilles semblables à celles de la joubarbe, mais un peu plus rondes, plus petites et moins longues et rampe sur le sol [= c'est une plante au port tapissant]. C'est la même plante qu'on appelle également Porcelane.) (p. 27)

Si on excepte la définition qu'en donne le Supplimento a'vocabolari italiani de Giovanni Gherardini (vol. VI, Milan, 1857, s.v.), ce passage du Trattato degli orti constitue la seule et unique attestation de ce phytonyme dans toute la littérature italienne.

\subsection{Appio romano (« céleri romain ») (hapax lexical)}

Trovasi dell'Appio chiamato Romano, il quale ha la foglia larga, dolce, e gustevole più d'ogn'altro. Se ne ritrova in quelle spaziose campagne, rasente l'acque stagnanti nelle vallate che vi sono.

(On trouve aussi du céleri qu'on appelle romain, qui a les feuilles larges; il a une bonne saveur et, plus que toute autre variété, est agréable au goût. On en trouve dans les vastes campagnes romaines, sur les berges des étangs, dans les vallées de cette région.) (p. 33)

Ce passage constitue la seule attestation de l'appio ou prezzemolo romano.

\subsection{Carota fiamminga (« carotte flamande ») (hapax lexical)}

Darlo schietto [il letame] ai cappucci, alle lattughe, alle carote, pastinache e radici, e a tutte l'erbe che barbano addentro, come prezzemolo e radicchi e carote fiamminghe.

(Il faut l'épandre [le fumier] pur sur les choux, les laitues, les carottes, les panais, les radis et sur toutes les herbes qui produisent des barbes [du chevelu] comme le persil, la chicorée et les carottes flamandes.) (p. 22)

Il s'agit de toute évidence d'une variété éteinte de carotte. Sur la base de l'origine géographique évoquée par Soderini on pourrait songer à la «carotte d'Amsterdam », ou à la variété « Berlicum », ou encore à la « Blanche de Belgique ${ }^{45} »$. 
2.4. Cavolo nero crespo $=$ cavolo di Toscana, Brassica oleracea var. Acephala) (« chou noir de Toscane », « chou palmier ») / cavolo francese $=$ cavolo riccio, Brassica oleracea var. Sabellica L. $)(«$ chou frisé ») (néologismes lexicaux)

E doppo i tredici d'aprile si pianta il cavolo nero crespo, ed il Romano, ed il Franzese.

(Après le 13 avril on plante le chou noir, le Romain et le Français.) (p. 89)

\title{
2.5. Dragoncello Lombardo («Estragon de Lombardie ») (hapax lexical)
}

\begin{abstract}
Ne sono di due fatte, l'ordinario, che ha la foglia distesa e lunga, ed il Lombardo, che l'ha un poco più corta e intagliuzzata. E più si distende schiacciato a crescere su per il terreno.

(Il y en a de deux sortes, le commun, qui a les feuilles longues et régulières, et celui de Lombardie, qui les a un peu plus courtes et dentelées. Ce dernier a tendance à s'étaler au ras du sol.) (p. 100)
\end{abstract}

L'évocation de cette plante locale - ce que rappelle le déterminant géographique lombardo n'est pas aussi anodine qu'on pourrait le penser d'emblée. En effet, le dragone ou dragoncello comune (Artemisia dracunculus L.) était encore une espèce considérée comme exotique dans les jardins italiens en cette fin de Cinquecento ${ }^{46 .}$ On peut donc supposer que l'adjectif rajouté par Soderini soulignait une différenciation entre une essence propre à l'Italie et une autre fraîchement introduite. On pourrait même penser que le végétal dont il est question dans ce passage n'est pas du vrai estragon mais qu'il a été défini comme tel au vu de l'effet de mode engendré, comme cela arrive souvent dans ce domaine, par l'arrivée sur le marché d'un « article » nouveau.

\subsection{Erba del sole (hapax) / Erba massima (hapax) / Fior di San Marco (hapax) / Tazza regia (hapax) = girasole (« tournesol») (n.1.)}

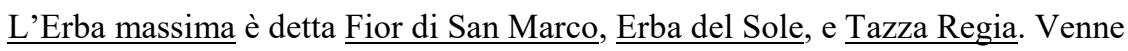
trasportato il suo seme dall'Indie da Mezzogiorno ; meriterebbe d'essere annoverata tra le piante grandi, se ella ciaschedun anno non venisse meno ; tanto s'innalza, ingrossa e cresce. Somiglia di nascita la pianta piccola di zucca [...] le foglie somigliano quelle delle zucche ma più nervose e tenaci, e con il gambo più lungo, ma più sottile, che mondo, e cotto ad uso degli asparagi, non è al punto disaggradevole. Il suo fusto ingrossa quanto il polso d'un braccio ordinario, crescendo talora all'altezza d'una picca e in cima fa una boccia grande [...] questa matura s'apre e dilata, avendo dentro a modo d'un vespaio i semi che sono mondi
} 
come fagiuoli aovati di color bigerognolo, dentro con un tenerume, che mondandone quantità se ne fa grato savore, e del calcio di quella boccia monda se ne fa pezzi, che cotti nell'acqua si mangiano conditi come i carciofi cotti, ed ancora si friggono e non sono insoavi [...] Il suo fiore è giallo, volto sempre al sole.

(L'Erba Massima est appelée également [...] Sa graine fut introduite à partir des Indes, par la route du Midi. Cette plante mériterait d'être insérée dans la catégorie des plantes supérieures [ = arbrisseaux]) si elle ne disparaissait chaque année, tellement elle s'élève, grossit et pousse. A la naissance elle ressemble à une jeune pousse de courge [...] ses feuilles ressemblent à celles de la courge mais elles sont plus nerveuses et tenaces, sa tige est plus longue, plus fine, et, une fois ébouillantée comme les asperges, n'est pas mauvaise. La tige s'épaissit jusqu'à atteindre la grosseur d'un poigné d'homme de stature moyenne, et la taille d'une pique, au sommet de laquelle pousse une sorte de grosse boule $[\ldots]$ cette dernière, arrivée à maturité, s'ouvre et se dilate [= gonfle], laissant voir à l'intérieur une multitude de graines qui rappellent la forme d'un nid de guêpe. Ces graines sont d'un beige assez foncé, de forme ovale, comme des haricots. Une fois ces graines mondées, la partie interne est agréable au goût ; le calice de la boule, on le nettoie bien, on le coupe en morceaux et on le mange mariné dans de l'huile et du vinaigre, comme on fait avec les artichauts cuits à la vapeur, ou sinon on peut le manger frit, ce qui n'est pas mauvais non plus [...] Sa fleur est jaune, toujours tournée vers le soleil.) (p. 102)

Ces phytonymes vernaculaires désignant le tournesol constituent tous des hapax lexicaux. L'apparition des lexèmes en question est peut-être liée à des observations directes : rappelons en effet que le tournesol a un emploi exclusivement ornemental en Europe jusqu'au début du XVII siècle alors que chez Soderini on l'utilise à des fins alimentaires. La première description occidentale de la plante, qui est l'œuvre de l'espagnol Juan Fragoso, daterait de $1572^{47}$. Dans cet écrit, la plante reçoit, entre autres, le nom vernaculaire de Sol de las Indias. En 1577 Francisco Hernandez de Toledo décrit le «chimalacatl péruvien, que d'autres appellent "fleur de soleil' ${ }^{48} »$. Il est vraisemblable que le syntagme erba del sole cité dans le passage du Trattato degli orti constitue un emprunt au phytonyme espagnol.

La description qu'en a faite Olivier de Serres, datée officiellement de 1600, année de la première édition du Théâtre d'agriculture et mesnage de champs, pourrait être contemporaine de celle de Soderini ${ }^{49}$ : "Quelque sympathie a l'herbe-au-soleil avec la passe-roze, par monter fort hautement. C'est une espèce de heliotrophon, appelée aussi vire soli, d'autant que sa fleur regarde tous-jours vers le soleil, se tournant comme lui. Communément chaque pied ne produit qu'une fleur mais fort grande, car elle a plus de demi-pied de diamètre. Elle est ronde, plate, façonnée comme carde de montagne, environnée de feuilles jaunes hautement

\footnotetext{
47 Discurso de las cosas aromaticas, cité par Mendes Ferrão [2015 : 197].

48 Mendes Ferrão [2015: 197].

49 A partir de la péninsule ibérique, où il débarque à la fin des années 1570 (Mendes Ferrão [2015:197]), le tournesol, pourrait avoir atteint l'Italie et la France à peu près simultanément.
} 
colorées. Au milieu de la fleur s'engendrent ses graines, lesquelles en leur maturité, sont semblables à celles des courges, grandes, blanches et plates... ${ }^{50} »$.

\subsection{Fagiolo (di Soria, turchesco, romano, armeno) (hapax lexicaux)}

Soderini aborde la question des haricots en deux passages. Dans le premier il dresse une liste, accompagnée de force détails, de plusieurs variétés : de ces dernières, quelques-unes sont encore existantes, d'autres éteintes depuis longtemps :

Il fagiuolo si dee allogare in terreno grasso ben lavorato, e tanto il Manuale quanto il

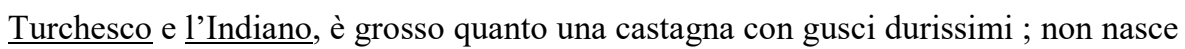
ne' nostri paesi, come d'un'altra sorte oltremodo grande, che viene d'Armenia. $\mathrm{Ne}$ sono degli Schiavoni e di Soria brizzolati, che quasi tutti nascono, ma non sono buoni per mangiare ma sono per bellezza. Buoni sono i Romani, grossi, senza negro e diventano cocendosi maggiori, con la punta del coltello levandolo loro.

(Il faut planter les haricots dans de la terre bien grasse et labourée en profondeur. Aussi bien le haricot "Manuale" que les haricots de Turquie ou d'Inde, ont la taille d'une châtaigne enveloppée dans une coque très dure. Ces haricots ne poussent pas sous nos latitudes, tout comme les haricots d'Arménie, qui atteignent des dimensions exceptionnelles. Il y en a également qui viennent du pays des Esclavons [ $=\mathrm{de}$ Dalmatie] et d'autres de Syrie, tout tachetés, qui ne sont pas des espèces comestibles mais ornementales. Les haricots romains sont aussi très bons, gros, sans œil et, à la cuisson, si on enlève ce dernier avec un couteau, ils gonflent.) (p. 107)

Parmi les espèces qui ont disparu de nos potagers nous pouvons compter ici le fagiuolo d'Armenia dont ce locus de Soderini constitue la seule attestation. Quant au fagiuolo di Soria, également hapax lexical, il s'agit d'une variété tout aussi inconnue des horticulteurs contemporains. Il n'est pas impossible que les fagiuoli di Sorana, dont il est question dans l'Epistolario ${ }^{51}$ de Giuseppe Giusti (1809-1850), puissent être identifiés avec la variété citée par Soderini. Le fagiuolo d'India est probablement le fagiuolo mungo verde (Vigna radiata $\mathrm{L}$.) (« haricot mungo »).

Deux dénominations vernaculaires s'avèrent particulièrement intéressantes en ce qu'il s'agit de régionalismes toscans : ainsi derrière le fagiolo turchesco faut-il vraisemblablement voir le fagiuolo turco grigio, dénomination toscane du fagiuolo di Spagna ( haricot blanc »). Cette nomenclature est répandue en particulier dans la Garfagnana; cela est d'autant plus significatif que le traité Degli orti fut rédigé à Volterra, ville du nord de la Toscane proche de cette région. Quant au fagiuolo romano («haricot romain »), cette variété, malgré l'épithète qui accompagne le premier élément du nom vernaculaire, n’est pas originaire de Rome mais 
également de Toscane (Florence et Arezzo). Ce dernier type de haricot est évoqué aussi un peu plus loin par Soderini, qui le qualifie également de fagiolo di Nizza:

Vanno ancora alti quelli di Nizza, detti anco $\underline{\text { Romaneschi, che non hanno alcun nero }}$ naturale, ma son scipiti più degli altri.

(Les haricots de Nice, qu'on appelle également Romains, s'élèvent aussi beaucoup.

Ils sont dépourvus d'œil, mais ils sont moins savoureux que les autres.) (p. 108)

Cette variété de légumes secs est également évoquée par Marco Lastri : «Si seminano ancora $\mathrm{i}$ fagiuoli detti romani in terreni peraltro freschi e nei divelti ${ }^{52} »$. Une telle abondance d'informations au sujet de ce légume n'est pas étonnante dans l'œuvre de Soderini, quand on sait son importance alimentaire dans l'Italie du $\mathrm{XVI}^{\mathrm{e}}$ siècle. La Péninsule était considérée comme le berceau de la culture de ce végétal d'où il serait passé en France en 1528, introduit par le chanoine Pietro Valeriano qui en avait reçu deux graines des mains du Pape Clément VII ${ }^{53}$.

\subsection{Passavelluto = Amaranto $(«$ Amarante ») (hapax lexical)}

L'amaranto vince tutti gli altri fiori di bellezza, manifattura e vivido colore ; chiamanlo i Franzesi Passavelluto, comecché sia di più bell'opera e più colorito del purpureo di quello che si dice Chermisi.

(L'amarante dépasse toutes les autres fleurs en beauté, grâce à son port et à sa couleur très vive. Les Français l'appellent Passevelours, parce qu'il est plus beau et que sa tinte pourpre est plus brillante que la couleur cramoisie.) (p. 34)

Le terme employé par Soderini semblerait être un emprunt au français passevelours dont la première attestation daterait de 1509 (passeveloux ${ }^{54}$ chez Jean Marie de Belges, Illustrations, éd. J. Stecher, t. 1, p. 215, TLFi, s.v.). Il indiquerait une variété ornementale de cette espèce, probablement cultivée à l'origine en France ${ }^{55}$, d'où elle aurait été exportée en Italie vraisemblablement dans la deuxième moitié du Cinquecento. Il pourrait s'agir d'une «plante

52 Corso di agricoltura, Florence, 1801-1803, vol. II, p. 17.

53 Toussaint-Samat $[1987: 45]$.

54 C'est sous cette même forme qu'on le retrouve chez Clément Marot : «Passeveloux, marguerites, lys et œillets » (Temple de Cupido, 1532, cité par Huguet V, p. 672). Plusieurs passages dans l'œuvre d'Olivier de Serres rappellent également la large diffusion de l'amarante dans les jardins français de la renaissance et de l'âge baroque : «Voici une fleur qui ne sent du tout rien non plus que les précédentes, c'est le passe-velours, dont la seule beauté, avec raison, le faict loger au jardin en lieu apparent. Elle est fort belle à voir, faicte en pyramide, de couleur incarnat esclatant : et qui plus est, séchée, ne descheoit que bien peu de son lustre. Qualité qui a faict ceste plante estre par d'aucuns appelée, passe-velours immortelle [...] Passe-velours branchu : c'est une autre sorte de passe-velours, s'eslargissant en brancheage ; dont il porte l'épithète de branchu: au contraire du précédent, qui s'en monte droictement en une seul tige. On l'appelle en latin, amaranthus maximus " (Olivier de Serre, Théâtre d'agriculture et mesnage de champs, Capitani 1996:887-888); "Quant aux œillets d'Inde, appelés aussi de Turquie, l'on ne le tient au jardin que pour la couleur, qui est belle, ressemblant au velours orangé »(Olivier de Serre [1996: 884]).

55 Le genre Amaranthus est originaire des régions tropicales. Il est donc plus probable que ses représentants aient été d'abord acclimatés en France, plutôt qu'en Italie. L'amaranthus caudatus L., originaire de l'Inde, était très populaire dans les jardins français de la fin du XVI siècle, où il était connu sous le nom de queue-de-renard (Pizzetti [1998: 35). On appelle également l'amaranthus caudatus amaranthus paniculatus. Si on considère que ses inflorescences ont été qualifiées de pannocchiute par Soderini quelques lignes plus haut, il est possible que ce soit cette variété dont il est question dans le passage en question. 
à fleurs rouges, de la famille des Amarantacées, du genre Celosia [du gr. kêlos «brillant»], proche des amarantes (amaranthus) également appelée Amarante crête-de-coq ou Célosie crête-de-coq (Celosia cristata $)^{56}$. Le TLFi précise qu' « on cultive fréquemment sous le nom de Passe-Velours une sorte d'Amarante dont l'inflorescence est faite d'une masse plate, portant d'innombrables petites fleurs ». Cette dernière variété aurait été vraisemblablement d'origine américaine, comme tous les cultivars décoratifs modernes. Qu'il s'agisse de la première ou de la seconde des deux plantes citées ci-dessus, le passage du Trattato degli orti pourrait en constituer le terminus ante quem et un élément fondamental pour dater son introduction dans la Péninsule. Dans tous les cas, le témoignage de Soderini est un indicateur de première importance de l'attention portée par l'auteur à des espèces auparavant inconnues en Europe occidentale ${ }^{57}$.

\subsection{Pinchero (d'Inghilterra) $=$ equiseto / coda cavallina [« prêle (d'Angleterre) »] (néologisme sémantique)}

\footnotetext{
Abbino di qua e di là in su li orli per lunghezza, e così gli altri andari più piccoli in traverso, spallierette basse di timo di levante, d'isopo, viole e abrotano e pincri d'Inghilterra e simili.

(Il faut que [les carrés de plantations] soient [...] longés par des bordures de thym oriental, hysope, violettes, armoise, prêles d'Angleterre et d'autres herbes du même genre. On fera pareille chose pour ce qui est des allées perpendiculaires, encore plus petites.) (p. 10)
}

La seule autre attestation du phytonyme vernaculaire pinchero est dans le Vocabolario di agricoltura de Canevazzi et Marconi (1892, s.v.) : "'Pincheri dei fossi' : nome volgare dell' 'equisetum arvense' [...] 'pincheri dei legnaioli': nome volgare dell' 'equisetum hyemale' [...] 'Pincheri dei prati', nome volgare dell' 'equisetum sylvaticum' ».

\subsection{Semprevivo arborescente (Aeonium arboreum L.)}

Dans un passage que nous avons examiné plus haut $(\S 1)$ Soderini énumère trois espèces de joubarbe (semprevivo) : celui qu'il définit maggiore, le minore et la troisième espèce qu'il qualifie d'arborescente. Ce témoignage d'une dénomination binomiale aujourd'hui courante est la toute première de la littérature italienne.

Soderini mentionne également cette espèce de joubarbe dans le Trattato degli arbori: «L'ellera, i melagrani, i gelsomini, la mortella, la madreselva, l'arborescente semprevivo, e la periploca, benché di queste sorte ce ne sia taluna che non regge l'invernata, per l'estate

56 Plantefol, Bot. et biol. végét., t. 2, 1931, p. 582, cité dans TLFi, s.v.

57 Les amarantes ne sont pas cultivées en Europe avant le début du XVI' siècle (Pizzetti [1998]). 
verdeggiano $^{58} »$. Cette nomenclature vernaculaire constitue une simple variante de celle qu'on trouve déjà chez Pietro Andrea Mattioli ${ }^{59}$, semprevivo arboreo, d'où a été probablement puisée la nomenclature binomiale latine linnéenne (Aeonium arboreum, 1752). Il est tout de même remarquable que l'adjectif employé par Soderini ${ }^{60}$ ait été nettement préféré dans la littérature botanique vulgaire des siècles suivants, au détriment justement du déterminant choisi par son plus illustre confrère siennois. C'est le cas par exemple d'Ottaviano TargioniTozzetti qui, dans ses Istituzioni botaniche (1802, vol. 2, p. 419) évoque justement le semprevivo arborescente, citant le phytonyme existant chez Mattioli seulement comme un synonyme secondaire. Le semprevivo arborescente est une espèce sous-arbustive à port érigé qui peut atteindre les deux mètres de haut. Elle est originaire du Maroc. Il se peut que les mentions retrouvées chez Mattioli et Soderini représentent les toutes premières références à une acclimatation de la plante en Europe occidentale ${ }^{61}$.

\section{Terminologie technique agronomique et horticole}

Plusieurs dizaines de termes techniques décrivent, dans le Trattato degli orti, des objets qui constituaient le quotidien de n'importe quel professionnel qui, au XVI siècle, s'attelait à la lourde tâche d'aménager le sol d'une exploitation ou d'un domaine. Ainsi en est-il de l'opération de transplantation des arbres : Soderini nous recommande de l'effectuer en faisant bien attention à ne pas enlever la «motte de terre accrochée aux racines de l'essence à déplacer ». Cette dernière est qualifiée de $\operatorname{pane}^{62}$ (di terra) (néologisme sémantique) :

Deesi da una parte del giardino che paia più appropriata, overo in un procinto di esso, che sia separato con muro, o con gran fossa o argine, piantar il salvatico d'arbori da ragia, come pini, abeti, larici [...] e andare per le piante in quei lati ove sian piccole, e cavarle con il loro pane, ed alla luna d'ottobre in luogo conforme al loro paese porle in buoni lavori di divelto ben sotto, ma non poi tanto addentro, e riguardarle dal bestiame.

(À l'endroit du jardin que vous estimerez être le plus approprié, ou bien dans un lieu proche de celui-ci, séparé éventuellement par un mur, un fossé ou encore un remblais de terre, il est conseillé de planter un conifère explanté dans la nature,

\footnotetext{
58 « Le lierre, le grenadier, le jasmin, la mortelle, la joubarbe arborescente et le chevrefeuil, bien qu'il y en ait des espèces qui ne passent pas l'hiver, pendant l'été restent bien verts » (p. 208).

59 Discorsi, vol. IV p. 1178.

60 La nomenclature Semprevivo arborescente serait déjà employée par Ulysse Aldrovandi (1522-1605) dans son Herbario (vol.VI, fo 38, p. 85), actuellement conservé dans le fonds manuscrit de l'Université de Bologne. Faute de datation précise de ce recueil, il est très difficile de se prononcer sur l'antériorité chronologique de cette dernière source par rapport à l'œuvre de Soderini. Les deux écrits sont à considérer à peu près contemporains. A ce sujet voir Biancastella, Ubrizsy Savoia \&Tosi [2003:245]

61 A ce sujet voir Larousse des plantes, p. 76

62 Pane (di terra) est très courant dans le Trattato degli orti : «Le felci traspiantatene con il loro pane, tuttoché non faccian seme, scavate sotto con tutte le radici e suo pane di terra [...] s'attaccheranno. Nate poi, e ristrette bene insieme, posson servire per ispallierette ». («Bien que les fougères n'engendrent pas de graines, une fois explantées avec une motte de terre accrochée aux racines, s'enracineront bien. Une fois qu'elles seront sorties de terre, serrées les unes contre les autres, feront de bonnes bordures de petites dimensions ») (p. 110); voir aussi dans cet article $\S 1$, (s.v. aovato).
} 
comme par exemple des pins, sapins, mélèzes [...] il faut aller chercher les arbres quand ils sont petits, les explanter avec une motte de terre encore accrochée à leurs racines, et, à la lune montante d'octobre, les replanter dans un coin du jardin qui s'approche le plus de leur lieu de naissance; il faut creuser la terre bien en profondeur, mais pas trop non plus, et les protéger de la dent des animaux domestiques.) (p. 19)

Ce terme technique est également employé par Bernardo Davanzati dans son traité d'agriculture intitulé De la coltivazione toscana delle viti e d'alcuni arbori ${ }^{63}$ (Florence, Giunti, 1600) : "L'ulivo vuole essere piantato con buon pane, in buono fosso o formella ${ }^{64}$. » D'autres sources, toutes toscanes, ont également conservé ce lexème. Ainsi en-est-il par exemple de Francesco Redi : «Si procuri di averne un alberetto giovane e col suo pane, e lo metta in un vaso di legno e lo accomodi bene con la terra ${ }^{65} »$; ou encore de Cosimo Trinci : « Io dico che si lascino in vivaio [gli ulivi] per sei o sette anni, che tanto basta perché vengano più grossi d'un manico di vanga, e allora si scavino senza il solito pane con tutte le barbe staccate affatto dalla terra ${ }^{66} »$. Le fait que la quasi-totalité des témoignages proviennent de cette aire linguistique tendrait à témoigner de la nature autochtone, probablement populaire, $\mathrm{du}$ mot. Une confirmation en ce sens nous vient d'un petit ouvrage de dialectologie de Giambattista Giuliani, qui compte cette expression parmi celles qui expriment le mieux les « délices » du toscan : « Si traspone l'ovolo col pane della terra impagliato ${ }^{67} »$.

Dans le domaine de l'horticulture au sens strict argine ou costa (néologismes sémantiques) désignent les «murailles », c'est-à-dire les bords rehaussés qui longent de part et d'autre le sillon tracé par un instrument aratoire, un araire ou tout simplement une houe :

Seminansi [i cetrioli] in fondo o in su l'argine o costa di un lungo solco, ricoprendoli poco, lontani l'uno dall'altro un bracccio...

(On sème [les concombres] sur le fond ou bien au sommet des parois d'un sillon, espacés les uns des autres d'une brasse...) (p. 98)

63 Opere, vol. II p. 515. Malgré la date de publication, postérieure à l'époque de rédaction de manuscrits du Trattato degli orti de Soderini, les deux ouvrages doivent être considérés comme quasiment contemporains.

64 «Il faut planter l'olivier avec une motte de terre attachée aux racines, dans une fosse bien profonde ou alors en pot ».

65 «ll faut bien veiller à se procurer un jeune exemplaire, avec une motte de terre accrochée aux racines. Alors on pourra l'installer dans un pot en bois, en faisant attention à bien disposer la terre tout autour» (Lettere (avant 1698), éd. Florence 1825, p. 42).

66 «Moi, je suis de l'avis que [les oliviers] il faut les laisser dans la pépinière pendant six ou sept ans, c'est-à-dire le temps nécessaire à ce que leur tronc atteigne la taille de celle d'un manche de pioche; c'est à ce moment-là qu'il faut les explanter mais, contrairement à l'usage habituel, il faut leur enlever la motte de terre qui reste accrochée aux racines : que ces dernières soient complètement à découvert » (L'agricoltore sperimentato, Lucques 1738, p. 112).

67 « Il faut transplanter le jeune plant d'olivier avec une motte de terre accrochée aux racines, bien enveloppée dans de la paille » (Delizie del parlare toscano, Florence, 1880, vol. I, p. 178). La première définition lexicographique de ce terme technique date seulement de 1811 . Il s'agit de l'entrée du Grand dictionnaire françois-italien composto sui dizionari dell'Accademia di Francia e della Crusca ed arricchito di tutti $i$ termini proprii delle scienze e delle arti, de Francesco d'Alberti di Villanuova, vol. 2, p. 424 : « Pane, dicesi anche al mozzo di terra appiccato alle barbe di qualsiasi pianta, Motte qui tient aux racines des arbres ». 
Les termes correspondants dans la moderne terminologie agronomique sont parete (di un solco) ou porca. Le sens que nous avons donné ci-dessus découle aisément du contexte immédiat. Le choix de ces deux vocables par Soderini est imputable très vraisemblablement à la ressemblance établie entre ce système de mise en culture et les berges ( $" \operatorname{argini}{ }^{68}$ » en italien) artificiellement mises en place le long d'un cours d'eau pour protéger les terres attenantes des crues soudaines, ou encore un coteau, une montée («costa » en italien). Il s'agit, me semble-t-il, de mots appartenant au basilecte.

Du terme technique argine Soderini emploie à plusieurs reprises les diminutifs arginetto et arginuzzo. Du premier on trouve deux occurrences dans le Trattato degli orti, citées cidessous, une première à propos de la plantation de l'ail :

E quando han dato fuori con tre foglie [gli agli], appianisi l'arginetto del solco, e si pareggi il terreno.

(Quand trois feuilles [des plantes d'ail] seront sorties de terre, il faut aplanir la paroi du sillon, et niveler la terre tout autour.) (p. 29)

Le deuxième locus se référant à la culture de la laitue :

Si trapiantano [le lattughe] nelle prode degli orti poste a solatio, ovvero in $\underline{\text { arginetti }}$ fatti a posta nel piano.

([Les plants de laitues] se repiquent sur le pourtour des vergers, dans un endroit bien ensoleillé, ou bien dans des monticules de terre mis en place exprès à un endroit plat.) (p. 145)

Il s'agit, à notre connaissance, des seules attestations écrites de ce lexème. Un troisième passage a conservé le diminutif arginuzzo :

Deono esser trapiantati [i petonciani] quando son grandicelli in sugli arginuzzi de' solchi, o rigoletti dove corra l'acqua.

(Il faut repiquer [les aubergines] quand elles commencent à être bien développées, au sommet des murailles des sillons.) (p. 201)

On trouve le premier témoignage en italien de ce dernier dans un petit traité d'horticulture de Pietro Lauro, rédigé en 1545 :

Plinio nel libro 19 al cap. 4 dice : mescola il grassume con la terra tre piedi sotto, et distingui l'horto in piazze attorniandolo con arginuzzi et con solchi, per i quai vada l'huomo, et corra l'acqua. Arginuzzo adunque è un terreno elevato tra due solchi, nel quale si pongono i semi cuoperti, et chiamansi ne i campi porca, da porrigo latina parola, che significa porgere fuori ${ }^{69}$.

68 Ce lexème peut désigner également un quelconque terreplein construit par la main de l'homme.

69 « Dans le livre XIXe, chap. 4, Pline dit : il faut mélanger le fumier à la terre jusqu’à une profondeur de trois pieds. Divise le potager en différents carré de plantations, en l'entourant de billons et de sillons, à travers lesquels l'horticulteur puisse se déplacer et évacuer l'eau. On appelle donc arginuzzo une portion de terrain qui s'élève entre seux sillons, dans lequel on sème. S'il s'agit d'un champ on l'appelle porca 
L'œuvre de Lauro est une traduction de l'un des plus célèbres traités d'horticulture de la première moitié du Cinquecento, écrit en latin par Charles Estienne en 1536, le De re hortensi libellus. Dans ce dernier le terme d'origine est pulvinus :

Est igitur pulvinus areola terram elatam inter duos sulcos complectens, in qua semina obruuntur, quae in agris porca a porrigendo appellatur ${ }^{70}$.

Puisque le mot-source ne constitue pas un diminutif, il est fort probable que le lexème du texte-cible (arginuzzo) n'en soit pas un non plus. Il pourrait s'agir d'un terme lexicalisé propre au basilecte dont la connotation diminutive - ce qui est logique aux yeux des premiers locuteurs l'ayant utilisé, puisqu'ils y voyaient comme une berge de fleuve (argine) ou un quelconque terreplein en miniature - n'était plus perçue.

Il est alors très vraisemblable qu'arginuzzo ou arginetto ne constituent pas des dérivés d'argine mais que ce dernier représente une retro-formation lexicale à partir de ces mêmes diminutifs. Il est plus compliqué de dire si ces mots sont des nouveaux termes forgés ex nihilo par l'auteur du Trattato degli orti ou des lexèmes populaires circulant oralement depuis longtemps.

Un synonyme d'argine et de ses diminutifs (arginetto et arginuzzo) semble être le terme proda (néologisme sémantique). Ce vocable est employé dans deux passages dans lesquels il est question de la mise en culture de la laitue :

Si trapiantano [le lattughe] nelle prode degli orti poste a solatìo, ovvero in arginetti fatti a posta nel piano.

([Les plants de laitues] se repiquent sur le pourtour des vergers, dans un endroit bien ensoleillé, ou bien dans des monticules de terre mis en place exprès à un endroit plat.) (p. 145)

Ou encore des figuiers :

Ma è meglio lasciare $\mathrm{i}$ viali liberi, e che niuna aia dell'orto sia soggetta a uggia, sebbene vi comportino i peschi e gli albercocchi. E in su le prode e ne' canti i fichi, ma questi tuttavia usufruttano il terreno, e con la loro ombra, nuocono agli erbaggi. (Il vaut mieux que les allées restent à découvert [= non couvertes par des berceaux de verdure] et qu'aucun carré du potager soit trop humide, bien que les pêchers et les abricotiers s'y accommodent parfaitement. Et sur le pourtour ou dans les coins on peut planter des figuiers, même si ces derniers épuisent la terre et, à cause de leur ombre, sont nuisibles aux légumes.) (p. 19)

\footnotetext{
en italien, du latin porrigo, qui veut dire extraire, faire sortir » (Di Carlo Stephano le herbe, fiori, stirpi che si piantano ne gli horti, con le voci più propie et accommodate, In Vinegia, Appresso Vincenzo Valgrisi, 1545, p. 20).

70 Charles Estienne, De re hortensi libellus, vulgaria herbarum, florum, ac fruticum, qui in hortis conseri solent nomina latinis vocibus efferre docens ex probatis authoribus, Parisiis, Ex Officina Roberti Stephani, 1536, p. 40.
} 
L'interprétation sémantique de proda s'avère assez complexe. En effet, si on considère que Soderini rédige son traité en Toscane, on aurait tendance à voir dans ce technicisme l'élément qui a donné son nom à un système d'exploitation agricole, celui justement de la «sistemazione a prode » (ou a rivale), qui est particulièrement répandu dans cette région italienne $^{71}$. Il s'agit d'un « modo di sistemare il terreno (diffuso in Val d'Arno, in Val d'Elsa e nella valle dell'Ombrone), che viene suddiviso in campi rettangolari, detti tramiti, con baulatura in sezione trasversale, filari di viti lungo i lati maggiori e prode inerbite, intercalati con fossi per la raccolta delle acque ${ }^{72} \gg$. Cette technique, employée surtout pour mettre à profit des lots de terrain sis en pente assez raide, a disparu de la plupart des pays d'Europe occidentale mais elle est toujours bien vivante dans le centre de la Péninsule, région où l'étage collinéen occupe une portion conséquente du territoire. Sa large diffusion, un peu partout en Italie, jusqu'au début du processus de mécanisation de l'agriculture moderne, après 1945, est confirmée par son évocation même dans des textes plus proprement littéraires ${ }^{73}$. Le passage du Trattato degli orti constitue le seul et unique témoignage écrit du terme - ainsi que de l'opération agricole qu'il signifie - de la Renaissance. Par la suite les sources restent silencieuses jusqu'aux indications, par ailleurs bien avares de détails, laissées par Marco Lastri : «Avverta l'ortolano d'aggiustar bene le prode per potere in occasione di pioggia piantar a solca l'indivia e la lattuga vernina ${ }^{74} »$. La nature toscane de ces deux sources agronomiques ferait pencher pour un caractère strictement autochtone du mot.

D’un autre côté proda pourrait tout aussi bien posséder dans les passages cités un autre sémantème et désigner une "bande de terre occupant le pourtour d'une parcelle ». Ce serait justement le sens du mot dans les deux loci d'après le GDLI («porca esterna destinata alla coltivazione $^{75} »$, XIV p. 463). En l'absence d'éléments probants dans un sens comme dans l'autre, la question est destinée à rester sans réponse. Cavezzale, cavino et gambina, vocables apparemment synonymiques de proda et argine, désignant de façon sûre la «culture sur billons » (système a prode) ${ }^{76}$, feraient tout de même pencher pour la première hypothèse. Dans tous les cas, peu importe que le sens de proda et argine soit le premier ou le deuxième des deux signalés, étant donné que les deux modalités d'aménagement du sol avaient la même

\footnotetext{
71 De son lieu d'origine présumé dérive l'autre dénomination de sistemazione alla toscana. Une autre appellation, aujourd'hui moins usitée est colmata toscana, découlant de l'opération qui consistait à accumuler de la terre humide au centre d'un terrain (colmare) de façon à ce que toute l'eau s'écoule vers les bords (Fig. 1 et 2).

72 Enciclopedia Treccani en ligne, s.v. « proda ».

$73 \ll[\ldots]$ ma sembra che anche i prodotti della terra, qui si sia riusciti a chiuderli ordinatamente negli scomparti delle prode, come in una serie di colorita scacchiera » (Nino Savarese, Cose d'Italia, 1940, p. 177).

74 « L'horticulteur doit faire bien attention à bien disposer les billons, de façon à ce qu'il puisse planter l'endive et la laitue d'hiver même quand il pleut beaucoup » (Corso d'agricoltura, Florence 1801-1803, II, p. 65).

75 Le Manuale di agricoltura (sez. A, p. 156) en donne la définition suivante: «striscia di terreno larga 30-40 cm che si mantiene inerbita ma utilizzabile anche come vivaio o semenzaio ». Même si cette deuxième acception s'avérait être la bonne, le vocable employé par Soderini serait toujours à considérer un cas de néologie sémantique : en effet le premier témoignage cité par le GDLI est celui de Renato Fucini (1843-1921).
}

76 Ces termes techniques seront analysés ultérieurement. 
finalité, celle d'évacuer l'humidité en excès ${ }^{77}$ et permettre une meilleure culture des légumes du potager.

Ce même référent du monde agricole et horticole semble donc recevoir d'autres dénominations chez Soderini. Cavezzale (néologisme sémantique) par exemple ou encore caccino (hapax lexical) et gambina (hapax lexical) désignent également le système de culture sur billons, idéal, comme nous l'avons expliqué, sur des terrains en pente et très humides :

Il che [lo scolo delle acque] si procurerà con alzar la terra da un lato, perché scoli dall'altro [...] Ed è il vero di accomodar ciò a primavera e all'autunno; e a questo medesimo tempo è bene ancora terrazzare i campi che altri voglia disporre alla sementa, $\mathrm{o}$ acconciare e preparare per orti, perché si megliorano di terreno e si mantengon sani. Imperciò quei cavezzali o caccini (il cui terreno si dee condurre nei campi alla primavera) si arino di ottobre o novembre, affinché la terra bolla insieme e si scaldi ; e che ogni corteccia e qual'altra erba vi fosse marcisca, riducendo la loro terra in due gambine, o vaneggie sole, perché le assai piogge del verno posssan scorrer fuore, o vero in una sola quando il terreno fosse arenoso, o d'altra sorta che non patisca omore rompendoli bene, e sottilmente a marzo seguente, e dispensando la lor terra nel mezzo de' campi che patiscano d'acqua, e facendogli piani a modo di prati, con un poco di pendìo. Quelli dipoi che nell'autunno aranno bisogno di tal'opera, si rompano nel modo detto in febbraio o marzo precedente, lasciandogli così sin al novembre seguente, ed allora riarandogli, e portando la terra nei campi nell'ordine detto. Così questi per il caldo dell'estate, quelli per il freddo dell'inverno si ricuoceranno fermentando in modo che spolverizzeranno. L'arare grossamente i

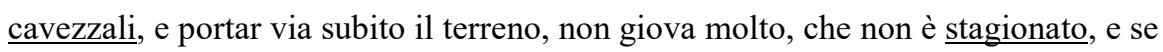
pure occorra portarlo dentro subito, arisi minutamente, ne mai quando sia zuppa la terra, e si rifaccia questo ogni tre o quattro anni.

(On évacuera l'excès d'eau dans la terre, en surélevant le champ d'un côté, afin que les eaux coulent dans la direction opposée [...] Il faut effectuer cette opération au printemps ou à l'automne ; c'est également à cette époque qu'il faut faire disposer les champs en terrasses pour les préparer aux semailles, idem pour les parcelles destinées aux potagers, pour en améliorer la qualité. Il faut donc labourer les cavezzali ou cavini (qu'on doit labourer également au printemps) en octobre ou novembre, afin que la terre ait le temps de s'améliorer et de désagréger en petites particules sous l'effet de la chaleur du soleil et que toutes les mauvaises herbes disparaissent. Il faut donc réaliser deux sillons pour l'évacuation des eaux pluviales qui s'y accumuleront pendant l'hiver [= deux billons] ou même un seul si le terrain est sablonneux ou d'une texture telle qu'il n'y ait pas trop d'humidité résiduelle. Il faut bien labourer ces parties du champ en profondeur au mois de mars qui suivra leur mise en place et jeter de la terre au milieu des parcelles qui souffriraient d'un excès d'eau et réaliser une pente assez douce, semblable à celle de certains pâturages. En revanche, les terrains sur lesquels de telles œuvres [d'assèchement] se rendraient nécessaires à l'automne, il faut les labourer en profondeur aux mois de février ou mars qui précèdent leur réalisation, puis les labourer une deuxième fois en

77 Cette bande herbeuse employée comme pépinière est toujours en pente et entourée de petits fossés de drainage. Il est vraisemblable d'ailleurs qu'il faut y voir une simple dérivation du système de culture a prode, qui était prévu, quant à lui, pour des plus grandes surfaces. 
novembre et seulement à ce moment-là rajouter de la terre comme nous l'avons expliqué ci-dessus. De cette manière, les uns fermenteront et se désagrégeront en menues particules [ $=$ se pulvériseront $]$ à cause des chaleurs estivales, les autres à cause du froid de l'hiver. Par contre, il n'est pas conseillé de labourer immédiatement les cavezzali et en enlever la terre, étant donné que cette dernière n'est pas encore bien fermentée ou alors, si on est vraiment obligés de l'enlever [pour la disposer tout de suite au milieu du champ] il faut la herser bien comme il faut, mais pas quand elle est humide. Il faut répéter cette opération tous les trois ou quatre ans.) (p. 21)

Dans le système de culture a prode le gambine ou vaneggie, appelées plutôt de nos jours prese, tramiti, presoni, représentent les «appezzamenti lunghi 80-120 m, larghi 15-33 m, leggermente baulati nella sezione trasversale ; lungo i lati maggiori, a circa $80 \mathrm{~cm}$ da una delle due scoline, sono presenti filari di vite maritata con aceri, olmi, gelsi. Si compongono anche di scoline, tubi di sgrondo di sezione trapezoidale, disposte longitudinalmente, che versano in capifosso situati alle testate dei campi. I cigli delle scoline sono rafforzati dalla proda, striscia di terreno larga $30-40 \mathrm{~cm}$ che si mantiene inerbita. La baulatura del tramite verso le scoline dovrebbe assicurare un rapido sgrondo delle acque superficiali ${ }^{78} \gg$. Dans le système $a$ cavalletto ou alla bolognese les mêmes éléments prennent le nom respectif de padiglioni (fette, pezze, morelli, canapine), alternant avec les cavalletti (strene) e scoline (Manuale d'Agricoltura, A, p. 146).

La description de Soderini correspond en France à la "culture sur billons ». Elle consiste à cultiver en rangées de petites buttes d'environ 15-20 centimètres de hauteur (6-8 pouces), préparées la saison précédente. Les buttes sont aplaties sur le dessus pour former une sorte de trapèze à pente douce évitant le ravinement en cas de fortes pluies. On plante sur le sommet du plateau. Ce système de culture et d'évacuation du trop-plein d'eau dans les champs a été abandonné en France après 1945.

Le terme technique cavezzale (plus connu dans l'agronomie moderne sous le nom de capezzagna ou cavedagna) désigne une «striscia di terreno inerbita e talora anche coltivata ; posta alla testata dei campi funge da strada e in qualche caso da invaso temporaneo per l'acqua in eccesso » (Ibid., A, p. 156). Capezzagna ou cavezzale seraient d'origine émilienne (GDLI II p. 929). La définition qu'en donne un dictionnaire dialectal de la fin du XVIII ${ }^{\mathrm{e}}$ siècle aide à définir la nature populaire de ces vocables, considérés comme des lexèmes propres aux patois de l'Italie du Nord-Est (Padoue et Venise): «Cavezzale del campo o caveagna. Acquaio. Solco a traverso al campo che riceve l'acqua degli altri, e ne la porta fuori. Cavezzale del fosso. Ciglione della fossa ${ }^{79} »$. Le synonyme caccino, que, sur la base du rapprochement étymologique avec le premier élément du couple lexical (cavezzale), il faut

78 Manuale di agricoltura, A p. 156.

79 Gasparo Patriarchi, Vocabolario veneziano e padovano, co' termine e modi corrispondenti toscani, Padova, 1796 , p. 67. 
vraisemblablement considérer comme une déformation de cavino ${ }^{80}$ ou capino, est inconnu des lexiques spécialisés. Il appartenait probablement au basilecte.

Quant à gambina, il s'agit d'un terme inconnu aux lexiques, également propre à la langue populaire, qui serait à rapprocher de gammitta (sic., calabr. centre-méridional), «canale di irrigazione, solco maestro destinato a ricevere le acque di scolo, solco di prosciugamento (di un campo) » (Cortelazzo \& Marcato [1998: 215]).

Dans tous les cas de figure que nous avons examinés dans les pages qui précèdent, l'auteur du Trattato degli orti n'oublie jamais de prendre en compte, avant toute œuvre d'aménagement et de mise en culture, le problème représenté par la surabondance d'eau qui pouvait mettre à mal les plantations. Cette question fondamentale était réglée, comme il a été montré ci-dessus, en disposant les terres sur des terrains en pente - qui constituent la plupart des espaces cultivables en Toscane, en particulier dans le nord de la région - et en mettant en place un ingénieux système d'évacuation des eaux en excès (système à prode).

Ce souci devait être constant dans l'agronomie ou l'horticulture de la Renaissance. Un terme comme sgorgatoio d'acque (hapax lexical), canale di scolo dans la moderne terminologie, c'est-à-dire un « canal d'évacuation de l'eau », ou un " orifice d'où s'évacue un trop-plein », en dit long sur les efforts prodigués par les professionnels de l'époque à ce sujet. Le problème devait s'imposer en toute son urgence surtout dans le cas des jardins suspendus, si fréquents dans les villas italiennes du $\mathrm{XVI}^{\mathrm{e}}$ siècle $^{81}$ :

Queste così fatte coltivazioni pensili si deono fare a piacimento secondo il potere, che fa di bisogno sia grande, perché vogliono buone volte, grosse, doppie di buon lavoro, con gagliarde spalle di muri grossissimi, e con catene di ferro se bisogni a traverso, e grandi sfiatatoi e sgorgatoi d'acque.

(Chacun pourra réaliser ces jardins suspendus, qui sont forcément assez imposants, selon son statut et sa richesse : en effet ces jardins nécessitent la mise en place de voûtes d'une taille importante, bien bâties, prenant appui sur des murs de soutènement très épais, fournis, si besoin il est, de grappes et tenons de fer, ainsi que de grands canaux [tuyaux ?] d'évacuation des eaux de ruissèlement.) (p. 25)

\footnotetext{
80 Cavino serait vraisemblablement dérivé de cavina (s.f.), que le Libro di Mattasalà di Spinello, texte siennois rédigé entre 1233 et 1243 , emploie dans le sens de «canal d'écoulement des eaux usées » ou encore "fossé longeant un champ destiné à évacuer les eaux en excès » ( $T L I O$ s.v.), le lien sémantique entre les deux étant ce mécanisme utilisé pour éliminer l'humidité excessive. Le fait que la première source nous ayant transmis le terme cavina soit toscane, tout comme les autres mentionnées par le TLIO, confirme indirectement le caractère autochtone et populaire du sodérinien caccino.

81 A ce propos voir Masson [1961 : 59 (jardins suspendus du Palais Ducal d’Urbino), 86 (Jardins suspendus de Palazzo Venezia à Rome)]. En ce qui concerne les systèmes de captation et de drainage des eaux dans les villas italiennes de la fin du XVI ${ }^{\mathrm{e}}$ siècle Lazzaro [1990 : 88] explique que plusieurs passages du Degli orti, dont celui-ci, rappelleraient de près des chantiers réels de l'époque, tel celui réalisé par Cosimo Bartoli pour le jardin de l'évêque de Cortona à Florence. A ce sujet voir aussi Lazzaro [1990 : 58-68].
} 
Le substantif sgorgatoio est dérivé de sgorgare 82 , «far defluire le acque (un canale), il liquame (una fogna) » dont la première attestation est dans un texte de la fin de la Renaissance connu sous le nom de Commedia intitolata "sine nomine" (1573) (cité dans GDLI XVIII p. 1000 n. 11).

Le français dégorgeoir, ayant le même sens que sgorgatoio (1788, TLFi s.v.) pourrait être un emprunt à l'italien, et témoigner d'une nette avance des jardiniers italiens de la Renaissance dans tous les travaux de nature hydraulique. La chose ne surprend pas quand on sait que les premières expériences en la matière datent dans la Péninsule du milieu du $\mathrm{XV}^{\mathrm{e}}$ siècle $^{83}$.

La même attention prêtée à la question du drainage transparaît derrière le verbe travoltare (l'acqua) (hapax sémantique), indiquant l'opération d' « évacuer l'eau en la faisant couler, par le biais d'un canal ». Etymologiquement l'expression signifie « détourner l'eau en l'orientant en dehors des cultures » (de extra + voltare, fréquentatif de volvere $)$ :

L'acqua sia solita traboccare o allagare gli spazi che contengono l'aiuole, déono essere dagli orli più alte di loro, perché più agevolmente vi facci l'entrata l'acqua che vi si avvia, e poi che l'assetata abbi satolle racchiuse, si possi nell'altre travoltare.

(L'eau ayant tendance à imbiber et même à déborder des carrés de plantations, sur les côtés la terre doit être à un niveau plus élevé qu'ailleurs, afin que l'eau puisse y entrer plus facilement dans un carreau donné et qu'on puisse plus facilement, une fois les plantations bien arrosées, la détourner vers celui d'à côté.) (p. 10)

Nous avons vu quelques lignes plus haut que les bandes de cultures, normalement horizontales, étaient disposées à flanc des collines (sistemazione a prode) pour permettre d'éliminer le trop-plein d'eau. Un terme comme resola (hapax lexical) pouvait au contraire désigner une aiuola ou porca ("carreau de cultures, bande de terrain cultivée ») située en terrain plus ou moins plat, situation plus commune dans le cas d'un petit lopin de terre ou un potager de ferme :

Le aie poi, o porche, o come si dice in Toscana l'aiuòle, e in Lombardia resole, che sono nei quadri distinte dai viottoli o piccoli sentieri, hanno a essere un po' più strette e lunghe per trasporvi l'erbe, cioè lunghe dodici piedi e larghe sei.

(Les carrés de plantations, qu'on appelle aiuòle en Toscane et resole en Lombardie, qui sont séparés les uns des autres par les allées, doivent être un peu plus étroits et disposés plutôt en longueur pour y faciliter le repiquage, c'est-à-dire qu'il faut qu'ils soient longs douze pieds et larges six.) (p. 10)

82 Cf. Sgorgata, «fuoriuscita di un liquido, in particolare acqua, la quantità di liquido sgorgata da una pompa idraulica » (Galileo Galilei, 1606, cité dans GDLI XVIII p. 1000).

83 A propos de l'œuvre novatrice de Léonard de Vinci dans la science hydraulique et la dynamique des fluides, voir Gille [1964 : 133, 140141]. 
D'après le GDLI (s.v.) resola serait un régionalisme lombard, ce qui prouve l'existence d'échanges entre les différentes régions de la Péninsule en matière d'agronomie. Le caractère régional de ce mot laisserait y voir un terme populaire, donc, par définition, assez répandu, malgré son statut linguistique d'hapax. L'attestation du terme dans le passage du Trattato degli orti montre encore une fois l'extraordinaire faculté de Soderini à conserver des lexèmes autrement perdus, ainsi que l'attention qu'il prête au monde de la campagne et aux mots qui en expriment ses aspects les plus caractéristiques.

Le Trattato degli orti nous renseigne aussi, comme on pouvait bien s'y attendre, sur l'une des opérations de base de l'horticulture, celle qui consiste à faire pousser des plants en pépinière pour les repiquer au printemps en pleine terre. Cette action est désignée par les verbes trasporre et trapiantare (néologismes sémantiques). Le premier est employé dans un seul passage où il est question de culture d'herbes, peut-être aromatiques :

E perché nell'autunno, e nella primavera principalmente, si seminano e si traspongono l'erbe negli orti, è cosa convenevole dividere le sue parti, e quelle lavorare nella primavera, che si vogliono seminare nell'autunno.

(À propos de l'ensemencement et du repiquage, qu'on effectue à l'automne mais surtout au printemps, il est utile de répartir les carrés de plantations et labourer au printemps ceux qu'on décide de semer à l'automne.) (p. 11)

Trapiantare est bien plus fréquent ${ }^{84}$. Il est associé également au repiquage d'herbes :

Le trapiantate [erbe] si sarchino più d'una volta, e rincalzandole, si dia loro nuovo letame fradicio. In trapiantando avvertiscasi (fatto il foro con un piolo) di non aggrovigliare le barbe, ma giù diritte mandarle, spuntandole sempre, perché piglian più forza a rimettere, e basta un sol dito svettarle. Aggravisi col piolo ben la terra addosso si che si faccia toccare per tutto, e sien rincalzate da ogni banda, e subito ch'elle sien trapiantate, a tempo asciutto s'annaffino, e se le si trapiantino nel secco l'estate, annaffisi prima il terreno assai, e smaltita l'acqua, si trapiantin qui. (Il faut sarcler à plusieurs reprises les herbes et légumes qu'on aura repiqués, les butter et rajouter du fumier bien fermenté. Quand on repique, après avoir fait un trou avec un piquet, il faut faire attention à ne pas emmêler leurs barbes [les radicules, le chevelu] mais au contraire les enfoncer dans le trou bien droites, en les coupant, afin qu'elles poussent avec plus de vigueur, en ne les laissant dépasser du sol que d'un petit doigt. Ensuite, il faut bien tasser la terre avec le piquet et rechausser tout autour du pied. Tout de suite après les avoir repiquées, quand il fait sec il faut les arroser, et, si on effectue le repiquage l'été, par temps sec, avant l'opération il faut bien arroser la terre et repiquer seulement une fois que l'eau aura été absorbée.) (p. 17)

Mais aussi de poireaux ou oignons :

I porri e le cipolle si potranno più presto trapiantare [in primavera], e tutte quelle che s'hanno a trapiantare si possono seminare tra quelle che restano sul terreno, e queste

84 En plus des passages cités ici voir supra les loci relatifs à argine et infra $\S 4$ - à propos de marcito (letame). 
si possono diradare e lasciarvele per ordine, che faranno come le trapiantate, $\mathrm{e}$ massimamente ne' tempi dell'estate, la lattuga. Si possono ancora seminare mescolatamente più sorte d'erbe di quelle che s'hanno a trapiantare, fra quelle che non s'hanno a muovere d'in sul terreno, dove elle son seminate. Perché quando le vi saranno tutte nate, si leveranno quelle, e vi si lasceranno queste, come i porri, le cipolle, gli agli, le carote, le pastinache, le bietole...

(On pourra repiquer les poireaux et les oignons plus tôt [au printemps], et les disposer parmi les autres plantes qui ont déjà été mises en terre ; ces dernières, on pourra les espacer [...] elles se comporteront comme celles qu'on aura repiquées, surtout la laitue l'été. On pourra semer les herbes et légumes qu'on va repiquer parmi les plantes qu'on n'extraira pas de la terre où on les aura précédemment semées. Quand toutes les herbes seront sorties de terre, on en enlèvera certaines et on en laissera d'autres, comme par exemple les poireaux, les oignons, l'ail, les carottes, les panais, les bettes...) (p. 16)

Ou encore de l'oseille :

L'acetosa fa cespuglio di più cesti insieme, e si attacca e mantiensi trapiantata in buon terreno.

(L'oseille forme des pieds composés de plusieurs plantes serrées les unes contre les autres ; elle prend racine et pousse bien si on la repique dans de la bonne terre.) (p. 27)

Le sens « repiquer » (un légume du potager, après l'avoir fait pousser dans une pépinière) est inconnu au GDLI (XXI p. 237 n. 5), qui ne donne que celui de «transplanter des arbres ». Marco Lastri a conservé le substantif dérivé désignant l'opération horticole du repiquage : "Perché non si spighino [i cardi] il primo anno della trapiantatura $^{85}$ ". Le fait que ce sémantème nous soit connu par le biais de deux sources agronomiques toscanes pourrait permettre d'y reconnaître un régionalisme peut-être populaire.

Le français transplanter $^{86}$ (laitues) est attesté depuis 1528 (Huguet VII p. 315). A la différence d'autres technicismes analysés dans cet article, dont l'antériorité chronologique par rapport aux termes homologues français pouvait être l'indice d'une supériorité technique des cultivateurs italiens sur les collègues transalpins, ici ce phénomène ne se produit pas : la facile dérivation du terme technique à partir du latin (transplantare) laisserait supposer un caractère paneuropéen du verbe en question.

Le sémantème synonymique de trasporre conservé par le premier passage cité ci-dessus constitue un locus unicus dans toute la littérature italienne. Cette signification dérive du sens primaire «trapiantare (gli alberi)» («transplanter» des arbres), attesté depuis la première

85 Corso d'agricoltura (1801-03), vol. IV, p. 173.

86 Transplanter / trensplanter, référé à des arbres, nous sont connus par des témoignages écrits depuis le XII ${ }^{\mathrm{e}}$ siècle (TLFi s.v.). 
moitié du XIV siècle $^{87}$. Sur la base de l'origine toscane de la quasi-totalité des sources qui nous ont transmis le sens primaire de trasporre, il est très probable que le sémantème secondaire « repiquer des herbes » ait été à l'origine un régionalisme sémantique toscan, très vraisemblablement populaire. Ce fait est confirmé par Giambattista Giuliani qui, dans ses Delizie del parlare toscano (Florence 1880, I vol. p. 178) cite à plusieurs reprises l'opération agricole consistant à trasporre des rejets fertiles d'olivier: "Chiamansi ovoli quelle ciocchettine che si levano dal pedale di un vecchio ulivo, e son grosse quasi uova. Queste si sotterrano con cert'ordine in un terreno adattato, donde poi sorgono dei piantoncelli che si traspongon nei campi ${ }^{88} \gg$.

Les plants repiqués après avoir poussé en pépinière reçoivent également un appellatif spécifique, postime $\boldsymbol{e}^{89}$ (néologisme lexical), «transplant » en français. Postime est à l'origine un terme typiquement toscan, désignant un «insieme di giovani piante seminate e piantate in vivaio in vista del trapianto dopo il primo sviluppo » (GDLI s.v.). Toutes les autres sources écrites qui ont conservé ce terme technique de l'agronomie sont également toscanes, ce qui plaide, encore une fois, pour une nature populaire et autochtone du mot. C'est le cas par exemple de Giovanni Targioni-Tozzetti : «Vi si fanno ancora alcune semente e postimi d'erbaggi e frutti, in modo tale che non vi è un palmo di terreno infruttuoso ${ }^{90} »$; ou encore de Marco Lastri : «Si lavorano a vanga le terre, perché si stagionino e per poter piantare i postimi per l'inverno ${ }^{91} »$. Ce technicisme est encore en usage en agronomie et horticulture ${ }^{92}$.

Une autre opération courante dans le potager est celle du bouturage ${ }^{93}$. Il s'agit d'un type de multiplication végétative des plantes, consistant à détacher une partie du végétal et à l'enterrer. Elle donnera lieu à un individu en tout identique à la plante-mère ${ }^{94}$. Ce système,

87 Ce sémantème est déjà employé dans l'une des plus anciennes versions vernaculaires de l'Opus agriculturae de Palladius (1340), ainsi que dans la traduction florentine du traité d'agriculture de Pietro De' Crescenzi (vers 1305) (GDLI XXI, p. 237 n. 5).

88 «On appelle ovoli les bourgeons qui se forment à la base d'un vieil olivier. On les appelle comme cela parce qu'elles ont la taille et la forme d'un œuf. Il faut les mettre en terre [dans la pépinière] en procédant d'une manière bien déterminée. A partir de ces bourgeons pousseront de jeunes plants qu'après on transplantera en plein champ » (Delizie del parlare toscano, Florence, 1880, p. 328. Voir aussi ibid. p. 178 : «Si traspone l'ovolo col pane della terra impagliato ».

89 Voir aussi infra $\S 4$ le passage relatif à l'expression a buona digestione.

90 «On peut y effectuer de semailles et y repiquer des herbes et des légumes, de façon à ce qu'il n'y ait pas un seul pied de terrain inexploité » (Relazioni di viaggi in Toscana, 1768-1779, vol. I, p. 311).

91 «Il faut labourer les terres à la pioche, afin qu'elles soient plus friables et qu'on puisse y repiquer les légumes d'hiver » (Corso di agricoltura, 1801-1803, vol. II p. 65).

92 Ce lexème possède chez Soderini deux autres acceptions techniques : la première est celle de «terrain cultivé ou destiné aux cultures » : «Ancora fan bene di qua e di là delle strade del giardino le spallierette basse rase pari fuor del postime della possessione, messo dentro nei quadri » (Della coltivazione delle viti, p. 251); la seconde désigne une « greffe » à effectuer sur un quelconque arbre : « Si dovrebbe sempre dividere il postime che s'ha da fare, come che avendosi quest'anno sessanta piante o innesti, porne trenta all'ottobre e trenta al marzo » (Trattato dell'albori, p. 144). Ces différents sémantèmes, proches les uns des autres, confirment le caractère technique du mot postime, vraisemblablement d'origine populaire.

93 Sur la base du contexte et compte tenu des végétaux cités, il est peu probable qu'il s'agisse du marcottage, bien que ce soit justement ce dernier qui permet une multiplication de l'individu d'origine à partir des seules parties aériennes, normalement les rameaux (rami).

94 Voir Pizzetti [1998 : 978] ; Böhm [1992: 81-82]. 
très utilisé surtout en floriculture, est appelé talea ou bottura dans la terminologie italienne moderne. Soderini parle à ce propos de (impiantare) (con il) ramo (néologisme syntaxique) :

\begin{abstract}
Alcune [piante] ne sono che vengono dal seme, alcune dalla radice, altre col capo, altre con l'una e con l'altro, altre con la radice e col seme, altre con la corteccia, altre con la cartilagine [...] certe con la marza e con il ramo, come la ruta, l'origano, il timo ; ed il bassilico, il quale tagliano, come sia pervenuto all'altezza di un palmo, e rimette ; certe con la radice e col seme, come le cipolle, l'aglio, e i bulbi, e tutte quelle che hanno capo ; ma per quanto tutti i semi dell'erbe facciano, nella ruta la regola fallisce, perché, seppur nasce, tardissimamente viene innanzi, e perciò si impianta a rami.

(Certaines plantes se régénèrent grâce aux graines [se ressèment], pour d'autres il faut la racine, ou bien la tête, pour d'autres encore l'une ou l'autre, pour d'autres la racine et les graines, pour d'autres l'écorce, pour d'autres les bourgeons; il y en a qu'on peut greffer sur d'autres plantes ou qu'on peut multiplier par bouturage, c'est le cas de la rue, de l'origan, du thym, ou du basilic. Ce dernier, on le coupe quand il a atteint la taille d'une paume et il émettra de nouveaux rejets. Parmi les espèces qui se régénèrent par la graine ou bien si plantées avec la racine on trouve l'oignon, l'ail et les plantes à bulbe en général et qui se terminent en une tête. Bien que toutes les espèces herbacées puissent être multipliées par graine, en ce qui concerne la rue, cela ne marche pas : en effet, même si elle sort de terre, sa pousse est très tardive, c'est pourquoi on la multiplie par bouturage.) (p. 13)
\end{abstract}

Dans un passage cité plus haut ${ }^{95}$ la même modalité de multiplication est indiquée par l'expression a ramo.

L'un des passages cités ci-dessus à propos des verbes trasporre et trapiantare, a également conservé, seul parmi les innombrables textes d'agronomie et horticulture de la Renaissance, la trace du terme technique désignant la phase préparatoire au repiquage, celle consistant à spuntare (le barbe) (hapax sémantique), c'est-à-dire « raccourcir les racines », « ébarber » les plants élevés en pépinière. Du sémantème de spuntare conservé par ce passage du Trattato degli orti on ne connaît pas d'autre témoignage écrit. Le seul sens technique attesté de spuntare est celui de "couper au couteau les extrémités d'un légume, par exemple une asperge $»^{96}$. Le terme utilisé dans la culture moderne des asperges est sbarbare.

\title{
4. Terminologie agro-pédologique (sol et engrais)
}

Les néologismes ayant trait à la science des sols forment probablement la catégorie la plus importante parmi celles qui font l'objet de cette étude. Cette dynamique particulière est probablement le reflexe de l'épanouissement de cette discipline au milieu du XVI ${ }^{\mathrm{e}}$ siècle. Et ce sont généralement des spécialistes de jardins qui lui ont donné la plus formidable

95 Supra $\S 1$, à propos de l'adjectif arborescente.

96 Ada Boni : «Scegliete dei fagiolini di buona qualità, che non presentino l'inconveniente del filo, spuntateli e risciacquateli. » (Il talismano della felicità, Roma, 1984, p. 184, cité dans GDLI XIX p. 1066, n. 2). 
impulsion: L'architecture et ordonnance de la grotte rustique (La Rochelle, 1563), L'abrégé d'agriculture (La Rochelle, 1654), le Discours admirable de la nature des eaux et fontaines de la Marne (Paris, 1580) de Bernard Palissy (1510-1590) sont considérés par les historiens de la pédologie comme des textes fondateurs. Dans le dernier des trois ouvrages cités, Palissy touche le sujet essentiel de la fertilisation des terres, pour lesquelles il conseille d'employer, en plus des traditionnels engrais organiques, la chaux. Lui fait écho en Italie le médecin A. Bacci qui, dans Del Tevere, della natura et bontà dell'acqua e delle inondazioni (Rome, 1558) opte pour la méthode plus ancienne de l'inondation des terrains avant la mise en culture $^{97}$. L'œuvre de Soderini s'inscrit totalement dans cet intérêt généralisé pour cette « nouvelle » discipline.

Dans l'œuvre de Soderini, très nombreux sont surtout les adjectifs qui désignent une texture particulière du sol. C'est le cas du hapax sémantique debole, littéralement «faible », dans le sens de « pauvre en substances nutritives et en minéraux » :

[L'avena] non affatica troppo le terre, a tal che si può convenientemente seminare nelle terre stracche per farle riposare e seminarvi poi a tempo grano. Desidera tuttociò terreno grasso, e basta due volte ararlo. Nel magro ancora e debole viene, sebbene la non profitta molto a moltiplicare.

([L'avoine] n'épuise pas trop la terre, à tel point qu'on peut en semer dans les terrains déjà épuisés pour les laisser au repos, et par la suite y semer du blé. Toutefois elle demande une terre grasse, qu'il faudra labourer deux fois. Cependant, elle peut pousser également sur de la terre maigre et non fumée, mais dans ce cas elle ne se développe pas bien.) (p. 42)

Néologismes sémantiques sont également les participes passés stagionata (terra), indiquant un terrain «parfaitement sec et dont le soleil a amélioré la texture le rendant particulièrement facile à labourer» (GDLI, XX p. 39 n. 15 et p. 40) et fermentata (terra), employé pour caractériser un sol qu'on a ameubli ${ }^{98}$, en réduisant fortement la granulométrie de la texture pour en faciliter le labour grâce à l'ajout de fumier, ce dernier engendrant une élévation de la température (le contexte fait allusion au phénomène consistant à cuocer la terra, «cuire, réchauffer la terre $»)$ :

Adunque il terreno che sia destinato per seminarsi e piantarsi ad orto all'autunno, si lavori al mese di maggio, e quello che sia serbato a seminarsi a primavera, si lavori a ottobre e novembre ; e l'uno e l'altro si lasci, così smosso, riposare sin al suo tempo, perché con i freddi e brinate si ricuoca e stagioni, e per i caldi si prepari a buona

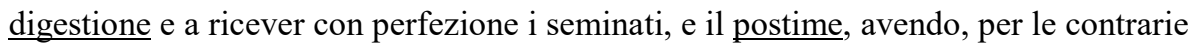
ragioni, tanto il caldo quanto il freddo, valor di cuocer la terra, e fermentata risolverla, disfar le zolle e ammazar l'erbe triste, annichilandone i semi.

97 A ce sujet voir Ubrizsy-Savoia [2000b : 384] ; Boulaine [1988 : 27-29].

98 Les termes techniques officiels utilisés dans l'agronomie italienne moderne pour désigner ces travaux précédant le labour proprement dit sont amminutare et amminutamento (Manuale di agricoltura A, p. 168). Pour l'opération de fermentare la terra voir aussi supra $\S 3$, à propos des termes trasporre et trapiantare. 
(La terre sur laquelle on voudra semer et planter à l'automne, il faut la labourer au mois de mai, et celle sur laquelle on voudra semer au printemps, il faut la labourer en octobre ou novembre ; une fois labourées, l'une et l'autre doivent être laissées au repos, jusqu'au moment des semailles : ainsi, les froids et les gelées d'hiver, ainsi que la chaleur de l'été, en amélioreront la texture en la fragmentant et la réduisant en petits morceaux. De cette façon la terre sera dans les conditions idéales être semée et les plants à repiquer, étant donné que aussi bien le froid que la chaleur, pour des raisons différentes, possèdent la même faculté de désagréger la terre, désintégrer les plus grosses mottes et même éliminer les mauvaises herbes, en tuant leurs graines.) (p. 11)

Les termes dont il est question ici devaient être courants dans le domaine agricole, si l'on en regarde le large usage qu'en fait Soderini. Ce dernier les emploie également dans son Trattato degli arbori ${ }^{99}$, ainsi que dans plusieurs autres loci du Trattato degli orti. Un témoignage de Marco Lastri est d'autant plus important que cet agronome est florentin comme Soderini, ce qui pousserait à voir des régionalismes sémantiques derrière les verbes fermentare et stagionare: "La terra non è più tanto sottoposta a fermentare e ribollire ${ }^{100}$ ». Egalement significative, et pour les mêmes raisons, s'avère une observation de l'agronome Giovanni Targioni-Tozzetti qui invite les professionnels à «senza confondersi in lasciar stagionar il terreno subito piantarvi la barbatella ${ }^{101} \gg$.

Le substantif digestione - dans l'expression preparare (un campo) a buona digestione - autre hapax sémantique, désigne, quant à lui, l'opération, préparatoire au labour véritable, de « frantumazione del terreno per rendere più facile l'assimilazione delle sostanze nutritive da parte delle piante» (GDLI IV, p. 406, n. 6). Ce technicisme sémantique dérive du sens « assimilazione delle sostanze nutritive da parte delle piante », attesté depuis le début du XIV siècle (Cresc. vulg., GDLI, ibid., n. 5). Si on rapproche le substantif digestione de l'adjectif digestito (infra) - «très fermenté » (le fumier) - cela laisserait supposer le recours à de l'engrais animal lors de ce processus de réduction des particules constitutives de la texture du sol.

Macero (hapax sémantique), associé également à la terre (terra macera), aurait vraisemblablement le même sens que les attributs qu'on vient de passer en revue. En effet son sens primaire, «pourri, moisi (en raison d'un excès d'eau)», ferait penser à un sol non pas imbibé d'eau, ce qui serait considéré comme un élément nuisible en agronomie, mais riche en

99 « Mettivi studio di far maneggiar simili lavori ai tempi buoni, e sieno asciutti, e giornata quieta, e la terra ben stagionata, fermentata, e purgata ».

(«Veille à toujours effectuer de tels travaux quand il fait beau et sec, sans grosses perturbations atmosphériques, et que la terre est bien friable, par l'actions conjuguée du soleil et du fumier, et pas trop humide», p. 140); « Nella maggior parte delle terre la megliore è quella della superficie, come più stagionata dal sole. » («Dans la plupart des cas, la meilleure partie du sol est sa couche superficielle, parce elle a été rendue bien friable par le soleil », p. 112).

100 «Ce n'est pas si facile que cela que de rendre la terre friable par l'action du soleil» (Corso di agricoltura, 1801-1803, II vol. , p. 21).

101 «[...] planter immédiatement les jeunes plants, sans attendre que la terre devienne plus friable » (Ragionamento dell'agricoltura toscana, Lucques 1759, p. 89). 
humus, ce dernier étant engendré par le processus de fermentation du fumier qui aurait été préalablement rajouté à la couche superficielle du terrain :

E vi si dee portar sopra la terra macera, e buona, più alta e fondata che si possa, ed ai tempi caldi mediante l'umore mantenervi l'umore.

(Et il faut la recouvrir de terre assez humide, bien fermentée et ameublie, d'une épaisseur aussi importante que possible, et par temps chaud l'arroser pour maintenir stable son degré d'humidité.) (p. 25)

Par ailleurs, l'auteur précise dans ce même passage qu'après les travaux préalables au labour, lors des chaleurs de l'été, il est conseillé de mélanger de l'eau à la terre, pour faire en sorte que son degré d'humidité reste constant (ai tempi caldi mediante l'acqua mantenervi l'omore). Cela confirme que l'état exprimé par l'adjectif macera n'est pas le fruit du même type de liquide. En outre, cette épithète relative à la terre est, nous semble-t-il, à relier aux adjectifs marcio et marcito qui caractérisent de façon sûre un fumier ayant dégagé beaucoup de purin suite à une fermentation très prolongée. Cela valide indirectement la signification du lexème qui nous occupe dans ce paragraphe.

Le sémantème qu'on vient d'examiner constitue une spécialisation technique ${ }^{102}$ à partir du sens « imbibé d'eau », « extrêmement humide », non attesté par les sources. Cette hypothèse pourrait recevoir une confirmation par la fréquente association à la terre du synonymique marcio (à partir du milieu du XIV ${ }^{\mathrm{e}}$ siècle, GDLI IX p. 780 n. 4). Or, depuis les premiers témoignages, ce dernier possède toujours des acceptions péjoratives comme dans ce passage de Boccace: «Dice l'autore che essi giacevano sopra il suolo della terra marcio, putrido, fetido e fastidioso, non altrimenti che il porco faccia nel loto ${ }^{103} »$. Malgré l'absence de sources autres que les passages du Trattato degli orti, il est fort vraisemblable que tous les déterminants qu'on vient d'analyser faisaient partie intégrante du langage agronomique. Ce silence est le signe de leur circulation au sein du basilecte exclusivement et, partant, de leur caractère populaire.

Spenta, néologisme sémantique extrêmement rare, caractérise une terre très riche en humus, contenant une quantité d'eau assez conséquente, mais non pas excessive. Dans tous les cas le taux d'humidité indiqué par cet adjectif devait être bien moins important que celui désigné par le susmentionné macero :

\footnotetext{
102 Cette restriction sémantique n'étonne pas quand on sait que le même Soderini emploie dans le traité Della coltivazione delle viti (p. 125) un autre terme composé de la même base de dérivation, par ailleurs encore vivant, marcita (s.f.). Ce dernier désigne un «tipo di prato, diffuso soprattutto in Lombardia e Piemonte e, meno frequentemente, nel Veneto, nel Lazio, in Umbria, sul quale, oltre all'irrigazione estiva (da aprile a settembre), si effettua quella invernale (da fine settembre a fine aprile) impiegando acque dotate di particolari caratteristiche termiche, in modo che esso possa vegetare tutto l'anno e fornire erbe utilizzate come foraggio » (GDLI, s.v.).

103 «L'auteur dit qu'ils gisaient sur le sol plein d'eau, recouvert de moisissures, putride, fétide et repoussant, comme des cochons se retournant dans la boue » (Guerri 1918: II, 193). Quant à marcioso, il a le sens de «fangoso, melmoso » (« boueux ») depuis la fin du XVIII ${ }^{\mathrm{e}}$ siècle (Opuscoli idraulici, 1829 - GDLI IX p. 782 n. 3). En français l'acception de l'adjectif correspondant est également péjorative : « terrains ainsi infectés de spores charbonneuses sont appelés : champs pourris ou champs maudits (GARCIN, Guide vétér., 1944, p. 214) (TLFi, s.v. pourri)
} 
Volendo seminarli [le conifere], procaccisi d'essi di tutte le sorte semente buone, e stagionate, e pongasi in buche ripiene di buon terriccio o in luogo grasso di buona terra spenta...

(Si on veut les semer [les conifères], il faut s'en procurer de bonnes graines, bien mûres, et les mettre dans des trous pleins de bon terreau ou dans de la bonne terre grasse et riche en humus) (p. 19)

Outre que du contexte immédiat le sens de spenta peut être déduit indirectement de celui de son contraire, cotta (terra), «fragmentée par l'action de la chaleur du soleil », attesté depuis le XIV ${ }^{\mathrm{e}}$ siècle ${ }^{104}$. Une description de la campagne toscane par Giambattista Giuliani confirme cette interprétation, en plus de définir cet emploi de spento comme typiquement régional : « Ha piovuto pochino; la terra non è ancora spenta; il sole la brucia, resta zotica del calore, indocile che non regge al lavoro ${ }^{105} »$.

Le sol le plus adapté au labour, en raison de sa friabilité superficielle qui permet aux instruments aratoires de pénétrer aisément en profondeur, est celui dont les cinquante premiers centimètres sont caractérisés par une texture assez fine. Les particules qui le composent sont assez réduites, généralement grâce au passage répété de la herse. Dans le Trattato degli orti cet état particulier de la terre est défini par les hapax sémantiques trito et sospeso $^{106}$ :

Desiderano tuti [i tipi di aglio] terreno grasso, dolce e piacevole a lavorarsi, ben trito

e sospeso, e più di color bianco, e cavato e smosso ben sotto, e senza letame ;

perciocché questo gl'incuoce e riscalda, rendendogli atti a marcirsi.

([Toutes les espèces d'ail] demandent une terre grasse, bien remuée et facile à travailler, bien hersée, plutôt blanche, qu'on aura labourée bien en profondeur et à laquelle on n'aura pas rajouté de fumier. En effet, ce dernier la désagrège en la réchauffant, favorisant ainsi la formation de moisissures.) (p. 29)

Ces sémantèmes sont attestés exclusivement chez Soderini (GDLIXIX p. 525 n. 3), qui les emploie également dans sont Dell'agricoltura e della coltivazione delle viti :

Bene è ancor cacciar nelle fosse sterpi, ricci di castagne, sarmenti o fascine che mantengono il terreno sollo e sospeso, da potervi meglio abbarbicare ${ }^{107}$. (p. 376)

Trito, qui veut dire littéralement «broyé », indique peut-être que cette réduction de la granulométrie du terrain n'était pas naturelle mais qu'elle était la conséquence directe d'un hersage répété. Cela est confirmé par la présence de l'adjectif crivellato dans le binôme trito e

104 Cf. Calce agricola spenta (« chaux hydraulique »), prodotto ottenuto per idratazione della calce agricola viva.” (Manuale di agricoltura, A, p. 195).

105 «Il n'a plu qu'un tout petit peu, la terre n'est pas encore imbibée d'eau, le soleil la brule, elle souffre des fortes chaleurs et la labourer est une tâche difficile » (Delizie del parlare toscano, 1880, I vol. , p. 423).

106 La terminologie agronomique officielle moderne parle de terrain amminutato ou sbriciolato (t.t., Manuale di agricoltura, A, p. 164).

107 Voir aussi, dans cette même section le passage relatif au couple adjectival crivellato et trito (infra). 
crivellato (infra), comme pour renforcer cette notion d'intervention humaine dans le processus, mais également par des sémantèmes assez proches et tout aussi répandus dans le domaine agronomique : ainsi trito a-t-il le sens de "dissodato », « labouré », chez Paganino Bonafé $\left(\mathrm{XIV}^{\mathrm{e}}\right.$ siècle - GDLI s.v. n. 3) ; la signification attestée chez Soderini pourrait n'être qu'une restriction sémantique à partir de cette dernière acception, liée plus particulièrement à la science des $\operatorname{sols}^{108}$. Cet emploi devait être propre au basilecte agricole. Il est improbable qu'il s'agisse de créations sémantiques purement "sodériniennes », attitude qui serait en contradiction avec sa faculté d'enregistrement de lexèmes et sémantèmes clairement populaires.

Il est remarquable que, dans le même passage, ce même sol léger soit qualifié de bianco, «blanc » (terreno [...] bianco), qui constitue l'énième cas de néologie sémantique. Les terres les plus meubles en surface sont en effet celles caractérisées par une couleur assez claire ou, tout du moins, non foncée. C'est ce genre de teinte qu'il faudrait voir derrière la description de l'horticulteur toscan, plutôt qu'une coloration franchement blanche. Cette dernière en effet désigne plutôt, en pédologie, des horizons argilo-crayeux ${ }^{109}$ qui, contrairement à la texture citée chez Soderini, sont très compacts et normalement inadaptés aux cultures ${ }^{110}$. On est en présence de la première attestation écrite de cet adjectif désignant une certaine composition agro-pédologique d'un sol. Comme dans d'autres cas de figures examinés dans cet article, l'auteur du Trattato degli orti s'avère être d'une importance capitale dans le cadre de la pédologie moderne. Son association des couleurs à la texture du sol est probablement la toute première du monde occidental $^{111}$.

Au couple adjectival trito - sospeso du passage ci-dessus fait pendant, comme fait allusion plus haut, trito e crivellato - ce dernier étant un hapax sémantique - dans lequel l'opération de fragmentation du sol en particules assez fines prend presque la forme d'un climax ${ }^{112}$ :

[L'amaranto] sementasi a primavera in terreno grasso, trito e crivellato.

(On sème [l'amarante] au printemps, dans une terre grasse, réduite en de tout petits morceaux.) (p. 34)

\footnotetext{
108 Voir aussi le sens « fin» (référé au sable) chez Girolamo Benivieni (avant 1545, GDLI s.v. n.4). Cf. stritolato (terreno) : «Il terreno smosso e stritolato dagli aratri e dalle zappe » (Francesco Patrizi da Cherso, 1529-1597, citation non datée, GDLI XX p. 381). Stritolato est également employé pour désigner la friabilité des roches calcaires (« La crosta stritolata dei monti », O. Targioni-Tozzetti, Lezioni di agricoltura, Florence, 1801, cit. dans GDLI, s.v. stritolato).

109 A ce sujet voir les tableaux officiels de référence de la couleur des sols (Munsell Soil Colour Charts et Standard Soil Colour Charts) ainsi que Baize-Jabiol [2011:91] et Lozet \& Mathieu [1998: 69].

110 La piètre qualité d'une terre blanche est confirmée par un passage contemporain d'Olivier de Serre : «La couleur ne suffit à telle instruction, bien que la noire soit la plus prisée de toutes, pourveu qu'elle ne soit marescageuse, ne trop humide ; car estant abreuvée, sera plustost de ceste-là que d'autre. La cendrée, la tanée, la rousse suivent après ; puis la blanche, la jaune, la rouge, qui ne valent presques rien » (Olivier de Serres, 1605, cité par le site mots-agronomie.inra.fr/mots-agronomie.fr/index.php/couleurs_des_sols). Voir aussi, dans cette même section, le passage relatif à renischio (infra).

111 Même un jardinier comme Bernard Palissy en France (1510-1590) pourtant céramiste de formation n'en parle aucunement.

112 Crivellato signifie littéralement "passé au tamis », ce qui exprime bien le processus de désagrégation de la terre poussé presque à l'extrême.
} 
Même le fumier (letame), l'unique engrais disponible au $\mathrm{XVI}^{\mathrm{e}}$ siècle pour augmenter la fertilité des sols et, par conséquent, directement lié à la texture pédologique de ces derniers, est accompagné de plusieurs déterminants qui en spécifient la composition. Dans le passage qui suit, notre horticulteur aborde la question, fondamentale pour les professionnels, de la fermentation des excréments animaux employés pour fumer la terre. Le fumier très fermenté, que Soderini qualifie de stagionato ${ }^{113}$ (GDLI XX, p. 40 n. 1) o digestito ${ }^{114}$ dans le locus en question, - il s'agit de tas de fumier qui reposaient pendant de longues semaines dans des entrepôts à l'abri du soleil de façon à ce qu'ils produisent une certaine quantité de liquide (liquame) - est considéré comme le seul à même d'avoir des effets positifs sur la production, celui n'ayant pas subi des processus de ce genre (crudo, «frais », hapax sémantique) étant franchement déconseillé :

Diasi agli orti [il letame) stagionato e digestito bene, perché il crudo non gli gioverà tanto.

(Il faut rajouter à la terre du potager [du fumier] bien fermenté, parce que le fumier trop frais lui sera plutôt nuisible.) (p. 23)

Ces épithètes techniques, dont on ne connaît pas d'autres attestations « anciennes » en dehors de celle de Soderini, se sont tout de même perpétuées dans la littérature agronomique des siècles suivants, comme on peut en juger par la lecture de quelques-uns des textes les plus célèbres. C'est le cas du Corso di agricoltura de Lastri (1801-1803, p. 239) : «Il moro richiede terreno fresco, sciolto e grasso, ovvero ingrassato con sugoso e stagionato letame $^{115} »$; de la traduction de Columelle effectuée par Benedetto del Bene (Columella volgarizzato, 1850, p. 36) : «Due letamai ancor vi sieno, l'un in cui porre i concimi freschi e serbargli un anno, e l'altro da cui trarre gli $\underline{\text { stagionati }}^{116}{ }^{16}$; ou encore de Francesco Agostino Gera : « Ma in caso diverso basterà [per coltivare gli ananassi] terra di prato ed unirvi un terzo di letame di vacca bene smaltito e stagionato, et un sesto di terra sabbiosa ${ }^{117}$ ». (Nuovo dizionario ragionato di agricoltura, 1846, vol. 25, p. 205). Cette série de témoignages semble indiquer leur appartenance au langage spécialisé, de l'agriculture. Cela est confirmé par l'article du Vocabolario di agricoltura de E. Canevazzi et F. Marconi (1892, s.v.) consacré à l'adjectif stagionato : "Stagionato : detto del letame che matura o si lascia a maturare ${ }^{118}$ » (GDLI XX p. 39, n. 9). Ce dernier est encore bien vivant dans la terminologie horticole contemporaine, à en juger par la recommandation suivante d'une revue bien connue des

113 Voir aussi, dans cette section, le passage relatif à viscoso (infra).

114 GDLI IV p. 407. Digestito est un hapax lexical.

115 «Le mûrier demande un terrain frais, léger et friable, gras, ou alors enrichi en y ajoutant du fumier encore riche en liquide bien qu'on l'ait laissé reposer longtemps ».

116 « Il faut installer deux dépôts de fumier, dans le premier on stockera le fumier frais qu'on y conservera pendant un an, dans le second le fumier qui y aura déjà séjourné pendant un long laps de temps ».

117 « Dans le cas contraire [pour cultiver les plantes d'ananas] il suffira de la simple terre végétale, à laquelle on rajoutera un tiers de fumier de vache qu'on aura laissé reposer longtemps et qui se sera vidé de tout liquide, et un sixième de terre sableuse ».

118 « Stagionato : se dit du fumier qui mûrit [= qui reste longtemps dans un entrepôt] ou qu'on laisse mûrir ». 
cultivateurs amateurs : «Il letame ben stagionato è un ottimo fertilizzante per le piante da fiore $^{119} »($ Vita in campagna, X, fasc. 10, 1993, p. 13).

L'homogénéité géographique des sources, toutes toscanes, laisse penser à des régionalismes, vraisemblablement populaires.

Synonyme de stagionato et digestito est l'adjectif marcio $^{120}$. Cet attribut, qui désigne une substance «très imbibée d'eau », ou même «pourrie » et « réduite à l'état de bouillie » en raison d'un excès de liquides, indique de toute évidence un type de fumier laissé fermenté pendant un très long laps de temps :

E perciò conviene bene addentro lavorare la terra e ben sotto tritarla dando loro [ai carciofi] assai cenere per suo grassume, e del letame marcio.

(C'est pourquoi il faut labourer la terre bien en profondeur et la réduire en petits morceaux et leur donner [aux artichauts] de la cendre comme engrais et du fumier bien fermenté.) (p. 66) [et passim]

Marcito, participe passé de marcire («pourrir », «moisir »), en représente une variante, attestée dans les passages suivants :

\begin{abstract}
Se occorre seminarle in non troppo gagliardo terreno [le fave], si dia loro letame marcito assai.

(S'il faut les semer [les fèves] sur un terrain pas trop riche, il faut rajouter du fumier très fermenté.) (p. 103)

E trapiantando il porro giovine [...] e rincalzando bene la terra attorno, e tagliandovi appresso ogni volta che ne rimetta le foglie sintantoché non cresca più, non toccando però dove metta il tallo, e gettando tuttaviaché si taglino tra essi del letame marcito. (Quand on repique des jeunes poireaux [...] il faut bien rechausser la terre tout autour du pied, et couper les feuilles chaque fois qu'ils en rejettent jusqu'à ce qu'ils n'en fassent plus, en faisant bien attention à ne pas toucher la talle, et épandre du fumier bien fermenté.) (p. 220)
\end{abstract}

Dans l'un des loci cités ci-dessus Soderini faisait allusion aux bénéfices produits par un fumier bien fermenté (stagionato), en lui opposant les effets néfastes de celui rajouté bien frais au sol (crudo). Dans un autre passage, notre professionnel emploie un autre adjectif qui semblerait en être, comme on peut en juger sur la base du contexte, un parfait synonyme, spento. Il s'agit de l'énième hapax sémantique du Trattato degli orti :

Su per il terreno spargere del concime fresco, non spento o marcio in quantità. (Sur la terre épandre beaucoup de fumier frais, pas trop fermenté et plein de liquide.) (p. 69) 
A l'opposé du type de sol sospeso, trito et crivellato est celui que Soderini examine dans les passages qui suivent. Ce dernier, en raison de leur forte teneur en eau, doit être quelque peu drainé mais s'avère être, aux yeux du professionnel, une sorte d'ersatz du fumier aujourd'hui les jardiniers parlent de «terre végétale »-particulièrement utile pour enrichir en substances minérales des champs autrement moins productifs ; ce type de terrain est défini

succhioso, viscoso ou encore tenace :

Nei campi umidi e succhiosi, facciasi il colmo alto nel mezzo, per liberargli del troppo umore. Ed essendo tuttavia asciutti e sabbionosi, non occorre fossi, ma sì bene spesso rifondergli di buon terreno ; e la terra che si ha a portar sopra i campi, dei fossi o d'altri luoghi cavata, si lasci prima a stagionare cinque, o sei mesi, poi si porti sopra i campi, se sia viscosa, tenace e forte e grossa. Ma se sia sabbionosa e risolubile, dolce e leggiera ed asciutta, e che si sfarini dopo breve tempo, vi si potrà condurre [...] Se si incontrerà terreno che bagnandone una zolla leggermente con acqua, e provando con mano se è tenace, o vi s'attenga e resti come pece attaccata alle dita, e gettata a terra non si sparga risolvendosi in minutissime parti, sara segno manifesto di grassezza.

(Dans les terres humides et modérément imbibées d'eau [tempérées], il faut faire des billons [renverser la terre de façon à élever un tas de terre au milieu d'un terrain en pente], pour en évacuer l'excès. Quand les parcelles sont sèches et sablonneuses, les fossés de drainage ne sont pas nécessaires, par contre il faut remettre de la bonne terre, creusée dans des fossés justement ou d'autres lieux similaires, qui aura été laissée fermenter pendant cinq ou six mois. Alors on pourra l'épandre sur les champs, si elle est riche en humus, assez compacte et à grains bien gros. Par contre, si elle est sablonneuse et friable, se désagrégeant facilement et dépourvue d'humidité, bref si, en la manipulant avec la main pour tester son degré de compactage, elle y reste bien collée comme de la poix, et si jetée par terre elle ne se désagrège en de très petites particules, cela constituera un signe manifeste de terre bien grasse [riche en substances nutritives].) (p. 21)

È bene ordinare il letamaio dell'orto ove scolino l'acque e vi stagnino, e quivi inviare tutte le brutture a marcirsi, e stagionarsi, e sia in lato ascoso il più che si può al sole e ai venti, o si cuopra con tavole, o doppio paglione perché si mantengano succhiose.

(Il est conseillé d'installer le dépôt de fumier dans la partie du potager où s'accumulent et stagnent les eaux pluviales ; c'est ici qu'il faut jeter tous les déchets pour qu'ils s'y décomposent, et fermentent bien; il faut que ce dépôt se trouve dans un endroit le plus possible à l'abri des vents et du soleil, ou bien, faute de mieux, on peut le couvrir avec des planches ou avec de la paille mélangée à du fumier, afin que les déchets gardent un certain degré d'humidité.) (p. 22)

Viscoso et succhioso (néologismes sémantiques) indiquent donc tous les deux une terre très riche en humus. Le premier constitue la toute première attestation - et la seule jusqu'au $\mathrm{XX}^{\mathrm{e}}$ siècle - de l'un des concepts fondamentaux de la pédologie moderne celui justement de viscosité $^{121}$. Quant à succhioso, un deuxième témoignage écrit est chez Francesco Fulvio 
Frugoni : «Quando le piante son velenose, per quanto sia il terreno fertile e succhioso in cui si obbligano, sempre più velenose e più feroci divengono ${ }^{122} »$. Succhioso pourrait représenter un cas de spécialisation technique par rapport à succoso qui a le sens de "riche de sève " (un végétal) depuis 1565 (Pietro Andrea Mattioli, GDLI XX p. 487 n. 1) mais il est plus probable de n'y voir qu'une variante du synonymique sugoso, dont le sémantème « riche en humus » est connu depuis le début du $\mathrm{XV}^{\mathrm{e}}$ siècle (GDLIXX p. 514). Les adjectifs employés par Soderini, d'origine vraisemblablement populaire, ne sont pas seulement le signe d'une curiosité innée chez l'horticulteur de Florence envers tout phénomène naturel, y compris ceux liés à la pédologie au sens strict, mais ils en disent long également sur l'avance des Italiens par rapport à d'autres pays d'Europe de l'époque, dans le domaine de la science des sols comme dans d'autres. Pour s'en convaincre il suffit de songer à la première attestation écrite $\mathrm{du}$ déterminant français équivalent, juteux, «imbibé d'eau » (un terrain), qui n'est probablement pas bien antérieure au tout début du $\mathrm{XX}^{\mathrm{e}}$ siècle s'il est vrai que son emploi métonymique date seulement de $1904^{123}$.

Le dernier élément du trinôme utilisé dans le passage ci-dessus pour décrire cette terre très humide, tenace, « visqueuse, collante, qui adhère fortement », parachève le portrait de ce type de terre par l'emploi d'une épithète technique dont les spécialistes se servent encore de nos jours pour désigner une sorte de terreau à la texture très proche de celle de la glaise et qui colle à la main du pédologue qui la manipule pour en évaluer le degré de compacité. La « manipulation ${ }^{124}$ » constitue d'ailleurs encore de nos jours la seule méthode fiable à laquelle les géologues ont recours pour établir un plan de fondation d'un bâtiment. Comme dans le cas des adjectifs viscoso et succhioso, tenace montre bien les progrès effectués en Italie en cette fin de $\mathrm{XVI}^{\mathrm{e}}$ siècle et l'écart existant par rapport à des pays comme la France ${ }^{125}$, pourtant terre d'élection de la pédologie depuis l'Ancien Régime. La convergence lexicale entre le passage du Trattato degli orti et une glose technique relative à la notion de « ténacité » dans le manuel de référence des agronomes italiens modernes en matière de pédologie, le Manuale di agricoltura Hoepli, met en évidence le savoir-faire acquis dans la Péninsule : « Tenacità : è la

122 «Les plantes venimeuses, même si la terre dans laquelle on les dispose est fertile et riche en humus, ne peuvent que devenir encore plus venimeuses et dangereuses » (La vergine parigina, Venezia, 1676, vol. III p. 19, cité dans GDLI XX p. 482 n. 1).

123 «Fr. Sol, pré juteux. [P. méton.] Les premiers entrés ont marqué leur pas juteux sur le parquet, de la barrière aux patères et des patères aux bancs ; bientôt, un chemin de boue s'est dessiné dans le préau (FRAPIÉ, Maternelle, 1904, p. 51)» (TLFi, s.v.).

124 Pour le style employé dans les tests de texture cf. Baize \& Jabiol [2011: 111] : «S'il y a beaucoup d'argile : plasticité («pâte à modeler »), mais résistance forte au pétrissage (échantillon dur à écraser), le pâton colle un peu aux doigts, devient même très collant s'il est gorgé d'eau ; surface lisse à l'écrasement entre le pouce et l'index; il est possible d'en faire un boudin assez fin. [...] S'il y a beaucoup de limons : le pâton est très facilement malléable, mais n'est pas plastique. Toucher doux, onctueux, «talqueux », à l'écrasement entre le pouce et l'index, la surface est 'pelucheuse'«.

125 Les premières attestations françaises ne seraient pas antérieures au premier quart du XIX ${ }^{\mathrm{e}}$ siècle : TLFi s.v. $\mathrm{n}^{\circ} 1:$ « Vieilli. [En parlant d'une substance molle ou liquide] Qui adhère fortement, qui est de consistance visqueuse, très collante. Le cérat est plus ou moins tenace, àpeu-près comme la térébenthine, et plus ou moins mou et onctueux, selon les ingrédiens qui en font la base (Kapeler, Caventou, Manuel pharm. et drog., t. 1, 1821, p. 173). On examina le lourd véhicule. Il se trouvait embourbé au milieu d'une vaste dépression du sol dans une glaise tenace (Verne, Enf. cap. Grant, t. 2, 1868, p. 209). Première attestation : 1628 [éd.] « qui est adhérent, visqueux (par la pituite) » (Paré, Euvres, XX, 27, éd. J.-Fr. Malgaigne, t. 3, p. 145)» (ibid.). 
resistenza che il terreno offre alla penetrazione degli organi lavoranti ; essa dipende dal grado di coesione delle particelle, dal grado di umidità e dalla tessitura del terreno. Aumento col diminuire del volume delle particelle (per cui è massima nei terreni argillosi e minima in quelli sabbiosi. L'argilla con umidità superiore a $17 \%$ diventa plastica e con più del $20 \%$ è $\underline{\text { vischiosa }}$ e si attacca fortemente agli organi lavoranti » (Manuale di agricoltura, A, p. 73).

Par rapport aux sols analysés jusqu'ici la granulométrie des terres sableuses ou sablonneuses est totalement différente : les particules qui les composent sont beaucoup plus petites, avec des diamètres compris entre $20 \mu \mathrm{m}$ et $2 \mathrm{~mm}$. L'adjectif renistìo et le substantif renìschio sont chez Soderini les termes définissant ce genre d'horizons pédologiques. Le premier, néologisme sémantique, indique des terrains où le sable ne constituerait pas la seule composante minéralogique, ce dernier se trouvant mélangé à des éléments plus grossiers comme du petit gravier ${ }^{126}$. La référence aux pastèques, qui recherchent ce genre de terre, confirmerait notre interprétation :

Il cocomero desidera terra grassa, umida di buon fondo, ben concimata, né meno ama quella che sia un poco renistìa, se vi sia del letame fradicio mesticato.

(Les pastèques demandent une terre grasse, humide et profonde, bien fumée ; mais elle s'accommode également des terres légèrement sablonneuses, si on y mélange du fumier bien fermenté.) (p. 82)

L'emploi du même terme par le naturaliste florentin Giovanni Targioni-Tozzetti pourrait faire penser à un régionalisme populaire, comme le GDLI (s.v.) semble également l'indiquer : « La mortella ama molto i terreni renisti di pietra serena ${ }^{127} »$.

Quant au néologisme lexical reniccio, il semblerait être l'équivalent nominal de l'adjectif renistio, désignant pareillement une terre sablonneuse composée d'un petit pourcentage d'éléments plus grossiers ${ }^{128}$ :

126 Renistio $<$ renischio $<$ renicccio $<$ lat. araenaceus. La composition de l'adjectif $(<$ rena + suff. dim. - schio $=-i c c i o)$ ferait penser à une texture du sol qui ne serait pas composée de sable à $100 \%$. Il ferait référence très vraisemblablement à un «horizon sableux à structure particulaire » (Triangles des texture GEPPA ; voir Baize \& Jabiol [2011:228]). Il s'agit d'un type particulier de peyrosols ou lithosols. Cf. le terme arénites (it. areniti) désignant une texture intermédiaire entre le sable et le gravier (taille entre 1/16 mm et $2 \mathrm{~mm}$ ). L'adjectif employé par Soderini semblerait correspondre, dans la moderne terminologie pédologique, à sablonneux (sabulous en anglais), caractérisant des horizons composés à plus de $80 \%$ de sable grossier (de diamètre compris entre 200 et $2000 \mu \mathrm{m}$ ), avec des pourcentages variables de limon, d'argile et de sable plus fin (Lozet \& Mathieu [1998 : 404]). Selon le GDLI (XV p. 813) reniccio (adj.) veut dire « Che contiene sabbia e frammenti minuti di pietra » (depuis la traduction de l'Histoire Naturelle de Pline de Lodovico Domenichi, 1561).

127 « La mortelle aime les terrains composés de sable et de fragments de pierre blanchâtre. » (Relazioni di alcuni viaggi fatti in diverse parti della Toscana, Florence, 1768-1779, vol. V, p. 297).

128 Reniccio (s.m.) désignerait chez Giovanni Targioni-Tozzetti, selon le GDLI (XV, p. 813) « frammenti minuti di pietra, ghiaino » (« Très petits fragments de pierre, petit gravier ») : «Ripulita la mina, v'introduce nuovo spolverìo o reniccio di sasso con nuova acqua. » («Une fois nettoyée la mine, on y introduit du petit gravier et puis encore de l'eau» - Relazioni..., vol. VII p. 236). Cette interprétation est indirectement confirmée par plusieurs régionalismes gallo-romans : fr. arenasso, "gros sable, vilain sable», areneàs, «terre vague», areneux, a.pr. arenos, n.pr. arenous, « terre vague », arêne (Wall.), « menu sable, gravier au bord des rivières », a.fr. areine, " grès », morv. areine, «granit en décomposition, lequel forme un sable à gros grains », areine (Nièvre), « gros gravier ou granit tendre effrité » (FEW I 135 b, s.v. araena). 
Desiderano le cipolle come gli agli bianco terreno, rosse, leggeri e non forte, renischio grasso di sua natura, e anche ingrassato con artifizio di letame.

(L'oignon et l'ail ont besoin d'une terre blanche ou rouge, légère, pas trop compacte, ou encore de sablon gras ou enrichi grâce à un apport de fumier.) (p. 83)

Reniccio est le résultat de l'ellipse de terreno dans le syntagme d'origine (terreno reniccio, c'est-à-dire "terrain composé de sable»), dans lequel il aurait été donc un déterminant adjectival (reniccio < lat. arenaceus). Différemment orthographié ce terme est assez répandu dans les documents littéraires des époques suivantes, dont un bon nombre sont de nature éminemment technique. Ainsi en est-il de Francesco Rondinelli : «Li seppellivano [i morti] mezzo miglio lontano dal lazzaretto [...] senza mettervi calcina, perché era renistio che si univa insieme, e non vi era pericolo si sentisse punto di malodore ${ }^{129} »$. Filippo Baldinucci donne, quant à lui la première définition de ce genre de matériau: «Renaccio, renischio, renistio : terreno simile alla rena o ripieno di rena ${ }^{130} »$. Le fait que la totalité des sources soient d'origine toscane ferait penser, comme dans le cas de renistio, à un régionalisme populaire.

En bon horticulteur qu'il est, soucieux de conserver la fertilité de ses cultures, Soderini se penche également sur le problème représenté par l'épuisement des sols suite à la mise en terre d'espèces végétales gourmandes en substances nutritives. Ces dernières usufruttano ou spoppano (« épuisent ») la terre, pour utiliser les mêmes termes que notre auteur. C'est le cas des câpres :

Poi fatti grandi [i capperi] da per loro stessi le [le erbe] spegneranno, usufruttuando e spoppando pur assai il terreno.

(Une fois sortis de terre et bien développés [les câpres] tueront les mauvaises herbes, épuisant en même temps la terre.) (p. 76)

Ou encore des figuiers :

In su le prode e ne' canti [piantare] i fichi, ma questi tuttavia usufruttano il terreno, e con la loro ombra nuocono agli erbaggi.

(Sur le pourtour du jardin ou dans les coins [on peut planter] les figuiers, en sachant que ces derniers épuisent la terre et que leur ombre nuit aux légumes.) (p. 19)

Usufruttare est un hapax sémantique (GDLI XX p. 597 n. 3) alors que spoppare est employé également dans le Trattato della coltivazione toscana della vite e d'alcuni alberi du florentin Bernardo Davanzati : «E tutti [gli alberi-tutori delle viti] si ponghino discosto dalle viti un

\footnotetext{
129 « Ils ensevelissaient leurs morts à une distance d'un demi mille du lazaret...sans rajouter de chaux étant donné que dans ce lieu la terre contenait un mélange de sable et de petit gravier et que donc cela ne risquait pas de sentir mauvais. » (Relazione del contagio di Firenze, Florence, 1634, p. 167)

130 «Renaccio, renischio, renistio : sol semblable à du sable ou qui se compose en partie de sable », Vocabolario toscano dell'arte del disegno, 1681, p. 134. Cf. Giovanni Targioni-Tozzetti (Relazioni di viaggi in Toscana (1768-1779), vol. V p. 107 : « Dipoi trovai terreno di collina, cioè renistio depositato o strati orizzontali sulle curve pendici del poggio. », " Par la suite je trouvai de la terre de colline, c'est-à-dire des petits gravillons mélangés à du sable, déposés en couches horizontales sur les pentes du coteau », cité dans GDLI XV p. 814).
} 
piè, e tuttavia secchi, e non mai verdi [...] perche i freschi spoppano il terreno e danneggiano troppo le viti ${ }^{131} »$. Le caractère apparemment régional de spoppare ${ }^{132}$, conjugué au fait que les deux verbes en question forment dans le premier passage une sorte de binôme, pourrait faire supposer que l'emploi d'usufruttare dans le Trattato degli orti est pareillement spécifiquement local.

\section{Conclusion}

Le traité Degli orti e giardini constitue un document essentiel pour nos connaissances en matière de jardinage et d'horticulture pendant la Renaissance italienne mais il se révèle d'une importance certaine précisément pour la langue spécialisée qui y est employée. Ou, pour être plus précis, c'est par l'intermédiaire des innombrables innovations lexicales qui caractérisent cette dernière qu'est éclairé tout un pan de l'histoire des techniques du XVI siècle en Europe : les néologismes du Degli orti donnent de précieuses informations sur la prééminence italienne dans l'art d'aménager jardins et potagers dans le Vieux Continent, même par rapport à la France, pourtant à la pointe des techniques horticoles depuis au moins un siècle et demi. Il suffit de songer au terme sgorgatoio ( $§ 3)$, parfait témoignage du haut niveau de la science hydraulique de la Péninsule, en avance d'environ deux cents ans sur le français dégorgeoir. C'est le cas également de l'emploi des adjectifs de couleur (bianco, §4) pour décrire la composition et la structure des horizons du sol, de certains qualificatifs comme succhioso [§ 4], « sol très riche en humus », qui précède de plus de trois siècles le français juteux, tenace

$(\S 4)$, « sol glaiseux et compact », antérieur de deux siècles par rapport au français tenace, ou encore de certains phytonymes vernaculaires (semprevivo arborescente $§ 2.10$ ) derrière lesquels il n'est pas impossible qu'il faille voir les premiers documents concernant l'introduction d'espèces végétales plus ou moins exotiques en Europe occidentale. Certaines nomenclatures vulgaires de végétaux (Erba del sole, § 2.6, Passavelluto, § 2.8) attestent au contraire l'excellence des jardiniers français dans ce domaine et une certaine influence sur leurs collègues italiens. Dans tous les cas, ces mots sont la preuve de liens culturels directs et durables et d'intenses échanges de savoirs entre scientifiques et techniciens des deux côtés des Alpes.

\footnotetext{
131 «Tous [les arbres-tuteurs] de la vigne doivent être plantés à la distance d'un pied des ceps et il faut qu'ils soient secs, non pas verdoyants...en effet quand ils sont verts ces arbres épuisent le sol et abîment les vignes. » (Bernardo Davanzati, Trattato della coltivazione toscana della vite e d'alcuni alberi, Florence, 1622, p. 29). Le traité de viticulture de Davanzati a été probablement rédigé peu après 1582 (voir Zaccaria [1987]).

132 Les deux sources qui ont transmis le terme sont florentines. En outre, ce verbe est utilisé dans autre locus par Soderini, quoiqu'avec l'acception différente de " indebolire la pianta madre » : « Nei luoghi piegati si dee scalzare di maniera che l'orlo della terra scavata si getti in su a rattener l'acqua fangosa che vi derivi dentro, e massime agli ulivi, e tagliando le barbicole, tanto più che spoppano l'albero ».

(«Dans les terrains en pente il faut déchausser le pied de l'arbre, de façon à ce que la terre qu'on vient d'enlever soit disposée du côté le plus élevée du tronc, pour faire barrage à l'eau boueuse qui pourrait remplir le trou. Cette opération est à effectuer surtout sur les oliviers, auxquels, entre autres, il faudra couper les radicules, parce qu'elles épuisent m'arbre », Trattato degli arbori, ed. Bacchi della Lega, p. 628).
} 
L'aspect le plus marquant du technolecte tel qu'il se dévoile à l'analyse du linguiste est son extraordinaire adhérence à la langue parlée dans la campagne toscane. Plus des deux tiers des termes examinés semblent bien provenir d'un fond lexical rustique qui doit bien remonter, dans la plupart des cas, au Moyen âge. Cela découle aisément d'une lecture même rapide non seulement de la liste de légumes du potager dont une courte liste a été dressée dans la deuxième section de cette étude mais aussi des vocables faisant partie d'autres catégories thématiques beaucoup plus spécifiques : c'est le cas de l'étude des composantes de la terre cultivable (pédologie) ou encore de la minutie et la précision terminologique presque modernes affichées dans la description des différentes parties des végétaux (physiologie végétale). Là aussi on entend très distinctement la voix du peuple du contado : c'est le cas de l'adjectif occhiuto, qui, en plus de son extrême technicité - il désigne le concept, très pointu en botanique, du verticille de la fleur - ne manque pas d'une certaine poésie, celle des rustres qui nomment ce qu'ils voient dans les prés lors d'un rare moment d'otium en l'affublant d'une épithète provenant du monde des humains. Les nombreux noms vernaculaires de plantes rappelant un œil (occhio) en représentent un témoignage irréfragable. Ce n'est d'ailleurs pas un hasard si tant d'écrivains célèbres ont redécouvert cette métaphore à l'époque moderne et contemporaine. On peut répéter les mêmes observations pour un autre adjectif relatif à la physiologie des plantes tel que pannocchiuto. Cet attribut, encore utilisé en botanique, et dont la forme panicolato doit remonter au moins au XIV ${ }^{\mathrm{e}}$ siècle, semble bien être puisé lui aussi à l'univers lexical des gens des campagnes. Or ces attributs sont encore bien vivants dans le technolecte botanique actuel. Encore plus significative est l'abondance de termes populaires qui font aujourd'hui partie intégrante du jargon des pédologues comme tenace ou viscoso. Une conclusion s'impose d'elle-même : un pont a été bel et bien jeté entre un savoir populaire et la science contemporaine, et ce fil rouge qui permet ces interconnexions lexicales c'est l'œuvre de Giovanvettorio Soderini. Ce dernier agit en tant que catalyseur et c'est probablement grâce à l'influence exercée par les écrits de cet horticulteur de Florence et à la reconnaissance qu'on lui accordait que les professionnels des âges suivants ont continué d'employer la même terminologie. Les sources citées dans les paragraphes consacrés à chaque lexème le montrent bien. C'est sûrement le legs le plus important que l'auteur du traité Degli orti e giardini ait laissé à la postérité. La terminologie italienne moderne des sciences de la vie et de la terre lui est entièrement redevable. Son œuvre que nous avons présentée ici est non seulement un texte fondateur de la science horticole moderne et de son vocabulaire mais elle permet également de regarder de près les principaux mécanismes qui président à la formation de n'importe quelle langue spécialisée ${ }^{133}$.

Des technolectes, y compris de ceux perfectionnés à l'époque contemporaine, la terminologie forgée par Soderini, possède, comme nous l'avons dit plus haut, la caractéristique essentielle qui est celle de la biunivocité lexicale et, partant, de la précision qui seule permet une 
description du monde de la Nature ne laissant aucune place à l'incertitude et au doute. C'est probablement l'une des raisons qui expliquent la fortune des néologismes du Degli orti jusqu'au $\mathrm{XIX}^{\mathrm{e}}$ ou même au $\mathrm{XX}^{\mathrm{e}}$ siècle: qu'on pense, pour s'en convaincre, à des technicismes tels que pane (di terra), trapiantare (§3) ou encore, dans le domaine de la pédologie, à stagionato $(\S 4)$, désignant un fumier qui a reposé très longtemps, et succhioso $(§ 4)$, indiquant une terre riche en humus, tous repris par les agronomes des siècles suivants.

Une dernière chose est à remarquer : le même processus de captation et conservation du patrimoine lexical des Anciens est observable dans d'autres textes scientifiques de divulgation. Ces travaux ont trait à des domaines différents et ont été rédigés à d'autres époques mais leurs auteurs se sont montrés tous également attentifs aux compétences pratiques de leurs prédécesseurs et tous ont su fixer sur la page écrite un savoir oral ancestral et la langue qui l'exprimait. Cela est vrai des traductions italiennes de l'Opus agriculturae de Palladius aux $\mathrm{XIV}^{\mathrm{e}}$ et $\mathrm{XV}^{\mathrm{e}}$ siècles ${ }^{134}$ comme de la production du zoologiste et médecin Francesco Redi ${ }^{135}$ (1626-1697). Ces convergences lexicales chez des auteurs si éloignés entre eux dans le temps et aux intérêts si divers permettent d'établir une méthode commune à l'œuvre dans la difficile tâche qui est celle de la vulgarisation de la science. Cela nous incite également à revaloriser les apports culturels des plus humbles aux connaissances des plus érudits.

\section{BibliogRAPHIE}

ANTONINI Annibale, 1762, Dictionnaire françois, latin et italien, contenant un abregé du dictionnaire de la Crusca et tout ce qu'il y a de plus remarquable dans les meilleurs Lexicographes, etymologistes et glossaires, qui ont paru en différentes langues, T. II, Lyon : les frères Duplain.

ATTLeE Helena, 2006, Italian Gardens. A Cultural History, Londres : F. Lincoln.

BaCCHi Della Lega Alberto, 1903, Opere di Giovan Vittorio Soderini. Il Trattato della cultura degli orti e giardini, Bologne : Romagnoli Dell'Acqua.

BAIZE Denis \& JABIOL Bernard, 2011, Guide pour la description des sols, Versailles : Quae.

BALdinuCCI Filippo, 1681, Vocabolario toscano dell'arte del disegno, Florence: Santi Franchi.

BARTOLINI Luigi, 1964, Poesie, 1911-1963, Padoue : Rebellato.

BECCARIA Gianluigi, 1995, I nomi del mondo, Turin : Einaudi.

Biancastella Antonino, Ubrizsy Savoia Andrea \& Tosi Alessandro, 2003, L'erbario di Ulisse Aldrovandi : natura, arte e scienza in un tesoro del Rinascimento, Milan : Motta.

134 Campetella [2016], Campetella [2016c].

135 Campetella [2016b]. 
Bianco Francesco, 2004, «Botanica e zoologia », in GrossmanN Maria \& RAINER Franz (éds.), La formazione delle parole in italiano, Tübingen : Niemeyer, 591-597.

BöHм Cestmir, 1992, Encyclopédie du jardinier, Paris : Grund.

Boulaine Jean, 1988, Histoire des pédologues et de la science des sols, Versailles : Quae.

Brochant de Villers Alexandre, Brogniart Alexandre, 1817, Dictionnaire des sciences naturelles, Paris- Strasbourg : F.G. Levraux- Le Normant.

Campetella Moreno, 2016a, «I neologismi tecnici dell’Opus agriculturae di Palladio: l'influenza della terminologia agronomica latina sui derivati romanzi », Lingue antiche $e$ moderne, $\mathrm{n}^{\circ} 5,85-118$.

Campetella Moreno, 2016b, «La nomenclature et le technolecte vernaculaires dans les traités de zoologie de Francesco Redi (1626-1697)», HAL-SHS: https://hal-univlyon3.archives-ouvertes.fr/hal-01416921

Campetella Moreno, 2016c, «Les traductions de 1'Opus agriculturae de Rutilius Aemilianus Palladius et la création de néologismes », in DE LEEMANS Pieter \& GoYens Michèle (éds.), Translation and Authority - Authorities in Translation, Turnhout: Brepols, 171-190.

Canevazzi Eugenio - Marconi Francesco, 1892, Vocabolario di agricoltura, Rocca San Casciano : Cappelli.

CaPitani Jean-Paul (éd.), 1996, Théâtre d'agriculture et mesnage des champs, Paris : Actes Sud.

ClaRICI Bartolomeo, 1726, Istoria e coltura delle piante che son pe '1 fiore più ragguardevoli e più distinte per ornare un giardino, Venise : Andrea Poletti.

Cortelazzo Mario \& Marcato Carla, 1998, I dialetti italiani. Dizionario etimologico, Turin : UTET.

D'Alberti di Villanuova Francesco, 1811, Grande dizionario italiano-francese composto sui dizionari dell'Accademia di Francia e della Crusca ed arricchito di tutti i termini proprii delle scienze e delle arti. Terza edizione italiana, Bassano : Giuseppe Remondini.

Dardano Maurizio, 1993-1994, «I linguaggi scientifici », in SERIANNI Luca \& TRIFOnE Pietro (éds.), Storia della lingua italiana, 3 vol., Turin : Einaudi.

DARDANO Maurizio, 2004, "Introduzione alla formazione delle parole nelle terminologie », in Grossmann Maria \& RAINER Franz (éds.), La formazione delle parole in italiano, Tübingen : Niemeyer, 575-580.

Davanzati Bernardo, 1600, De la coltivazione toscana delle viti e d'alcuni arbori, Florence : Giunti.

Del BeNE Benedetto, 1808, L'agricoltura di Columella volgarizzata, Verone: Giovanni Gambaretti. 
DionisotTi Carlo, 1967a, «Tradizione classica e volgarizzamenti », in DionisotTI Carlo, Geografia e storia della letteratura italiana, Turin : Einaudi, 125-178.

DionisotTi Carlo, 1967b, «La letteratura italiana nell'étà del Concilio di Trento », in Dionisotti Carlo, Geografia e storia della letteratura italiana, Turin : Einaudi, 227-254.

EsTiEnNE Charles, 1536, De re hortensi libellus, vulgaria herbarum, florum, ac fruticum, qui in hortis conseri solent nomina latinis vocibus efferre docens ex probatis authoribus, Parisiis : Ex Officina Roberti Stephani.

ETTY Thomas \& HARRIson Lorraine, 2016, Plantes anciennes, Chamalières : Artémis.

FORMENTIN Vittorio, 1994, «Dal volgare toscano all'italiano », in MALATO Enrico (éd.), Storia della letteratura italiana, IV, Il primo Cinquecento, Rome : Salerno, 177-250.

Frosini Giovanna, 2014, «Volgarizzamenti», in ANTONELLI Giuseppe et al., Storia dell'italiano scritto, II, Prosa letteraria, Rome : Carocci, 17-72.

GHERARDINI Giovanni, 1853, Supplemento a' vocabolari italiani, vol. 2, Milan : Giuseppe Bernardoni.

GiLle Bertrand, 1964, Les ingénieurs de la Renaissance, Paris : Hermann.

GimorRI Adriano (éd.), 1924, Raimondo Montecuccoli. I viaggi, Modène.

GiovanARDI Claudio, 2006, «Storia dei linguaggi tecnici e scientifici nella Romania: italiano », in GERHARD Ernst et al. (éds.), Romanischen Sprachgeschichte / Histoire linguistique de la Romania, Berlin-New-York : De Gruyter, II, t. 2, 2197-2211.

GiUliaNi Giambattista, 1880, Delizie del parlare toscano, Florence : Successori Le Monnier.

GrendLeR Paul Frederick, 1993, «Form and function in Italian Renaissance Popular Books », Renaissance Quarterly, n. 46, 451-485.

GrendLeR Paul Frederick, 1995, Books and Schools in the Italian Renaissance, Aldershott: Variorum.

Gualdo Riccardo \& Telve Stefano, 2011, Linguaggi specialistici dell'italiano, Rome : Carocci.

Guerri Domenico (éd.), 1918, Giovanni Boccaccio, Commento alla Divina Commedia e altri scritti intorno a Dante, Bari : Laterza.

LASTRI Marco, 1801-1803, Corso di agricoltura, Florence : Stamperia del Giglio.

LAUro Pietro, 1545, Di Carlo Stephano le herbe, fiori, stirpi che si piantano ne gli horti, con le voci più propie et accommodate, In Vinegia : Appresso Vincenzo Valgrisi.

LAzzaro Claudia, 1990, The Italian Renaissance Garden. From the Conventions of Planting, Design, and Ornament to the Grand Gardens of Sixteenth-Century central Italy, New HavenLondres : Yale University Press. 
Le Roy Ladurie Emmanuel, 1983, Histoire du climat depuis l'an mil. vol. II, Paris : Flammarion.

LERAT Pierre, 1995, Les langues spécialisées, Paris : PUF.

LIBRANDI Rita, 2003, «Il lettore di testi scientifici in volgare », in BOITANI Piero, MANCINI Mario \& VARVARO Alberto, Lo spazio letterario del medioevo. 2. Il medioevo volgare, vol. III. La ricezione del testo, Rome : Salerno, 125-154.

LINDLEY John, 1830, The Elements of Botany, with Glossary of technical Terms, Londres : Bradbury and Evans.

Lozet Jean \& MATHiEu Clément, 1998, Dictionnaire de Science du sol, Paris : Tec Doc.

LUCCHESI Laura \& BERTOCCI Stefano, 1984, Villa Il Giardino. Una dimora signorile nella campagna di San Salvi, Florence : Salimbeni.

MagalotTi Lorenzo, 1779, Operette varie, Venise : Pizzolato.

Maмmino Liliana, 1995, Il linguaggio e la scienza. Guida alla precisione del linguaggio, Turin : SEI.

MANDRessi Rafael, 2016, «Images et savoirs », in HiLAIRE-Perez Liliane, SimON Fabien \& THEBAUD-SORGER Marie, L'Europe des sciences et des techniques. Un dialogue des savoirs, $X V^{e}$-XVIII siècles, Rennes : PUR, 303-309.

Marazzini Claudio, 1993, Storia della lingua italiana. Il secondo Cinquecento e il Seicento, Bologne : Il Mulino.

MARTINi Ferdinando (éd.), 1932-1956, Giuseppe Giusti. Epistolario, Florence : Le Monnier.

Masson Georgina, 1961, Italian Gardens, Londres : Thames and Hudson.

Mattioli Pietro Andrea, 1563, I Discorsi di M. Pietro Andrea Mattioli Sanese ne i sei libri di Pedacio Dioscoride Anazarbeo Della materia medicinale, Venise : Vincenzo Valgrisi.

Mendes Ferrão José E., 2015, Le voyage des plantes et les grandes découvertes, Paris : Chandeigne.

Neveu Hugues, 1975, « Déclin et reprise : la fluctuation biséculaire. 1340-1560 », in DuBY Gorges \& WALLON Armand (éds.), Histoire de la France rurale. 2. L'âge classique des paysans : de 1340 à 1789, Paris : Seuil, 7-156.

Patriarchi Gasparo, 1796, Vocabolario veneziano e padovano, co' termine e modi corrispondenti toscani, Padoue : Stamperia Conzatti.

PizzetTi Ippolito, 1998, Fiori e giardino, Milan : Garzanti.

REDI Francesco, 1825, Lettere, Florence : Stamperia Magheri.

RiCHARDSON Brian, 1994, Print Culture in Renaissance Italy. The Editor and the Vernacular Text. 1470-1600, Cambridge : Cambridge University Press. 
Rondinelli Francesco, 1634, Relazione del contagio di Firenze l'anno 1630 e 1633, Florence : Giovan Battista Landini.

SALTINI Antonio, 1984, Storia delle scienze agrarie, dalle origini al Rinascimento, Bologne : Edagricole.

Santi Giorgio, 1795, Viaggi per la Toscana, vol. I, Viaggio al Monte Amiata, Pise : Ranieri Prosperi.

Savarese Nino, 1943, Cose d'Italia, Milan : Tumminelli.

Serianni Luca, 1993, « La prosa », in SERIANNI Luca \& TRIfONE Pietro (éds.), Storia della lingua italiana, I, I luoghi della codificazione, Turin : Einaudi, 451-524.

Serianni Luca, 2003, Italiani scritti, Bologne : Il Mulino, 2003.

Simon Fabien, 2016, «Quelle est la langue de la science : dire efficacement la "vérité" scientifique ", in Hilaire-Perez Liliane, Simon Fabien, Thebaud-Sorger Marie, L'Europe des sciences et des techniques. Un dialogue des savoirs, $X V^{e}$-XVIII ${ }^{e}$ siècles, Rennes : PUR, 257-267.

STEARN William Thomas, 1966, Botanical Latin, Londres : Nelson.

Targioni-TozzetTi Giovanni, 1759, Ragionamenti del Dottor Giovanni Targioni-Tozzetti sull',agricoltura toscana, Lucques : Stamperia Jacopo Giusti.

TARGIONI-TOzzetTi Giovanni, 1779, Relazioni di viaggi fatti in diverse parti della Toscana, Florence : Gaetano Cambiagi.

TARGIONI-TozzetTI Ottaviano, 1802, Istituzioni botaniche, vol. I, Florence: Stamperia Reale.

TARgioni-Tozzetti Ottaviano, 1809, Dizionario botanico, Florence : Guglielmo Piatti.

Tesauro Emanuele, 1669, Il cannocchiale aristotelico, Venise : Paolo Baglioni (1 ${ }^{\text {re }}$ éd. 1654).

ThEBAUD-Sorger Marie, 2016, «Les publics des sciences et des techniques : médiations / réceptions », in HILAIRE-PEREz Liliane, SIMON Fabien \& THEBAUD-Sorger Marie, L'Europe des sciences et des techniques. Un dialogue des savoirs, $X V^{e}-X V I I I^{e}$ siècles, Rennes : PUR, 333-353.

TousSAINT-SAMAT Maguelonne, 1987, Histoire naturelle et morale de la nourriture, Paris : Bordas.

Trifone Pietro, 1993, « La lingua e la stampa nel Cinquecento », in SERIANNi Luca \& Trifone Pietro (éds.), Storia della lingua italiana, I, I luoghi della codificazione, Turin: Einaudi, 425-450.

Trinci Cosimo, 1733, L'agricoltore sperimentato, Lucques : Marescandoli.

Trovato Paolo, 1994, Storia della lingua italiana. Il primo Cinquecento, Bologne: Il Mulino. 
UbRIZSY-SAVOIA Andrea, 2000, «Naturalistes », in KUSHNER Eva (éd.), L'époque de la Renaissance (1400-1600), III, Maturations et mutations (1520-1560), AmsterdamPhiladelphie : J. Benjamins, 434-459.

UbRIZSY-SAVOIA Andrea, 2000b, «La littérature des pratiques agraires », in KLANICZAY Tibor, Kushner Eva \& CHAVy Paul (éds.), L'époque de la Renaissance (1400-1600), IV, Crises et essors nouveaux (1560-1610), Amsterdam-Philadelphie : J. Benjamins, 375-386.

ZACCARIA Raffaella, 1987, «Bernardo Davanzati », in RomAnelli Raffaele (éd.), Dizionario Biografico degli Italiani, Roma, Istituto dell'Enciclopedia Italiana: www.treccani.it/enciclopedia/bernardo-davanzati_\%28Dizionario

\section{ABRÉVIATIONS}

DL $=$ DuBOIS Jean [et al.], 1973, Dictionnaire de linguistique, Paris : Larousse.

FEW = WARTBURG (Von) Walter, 1928-1966, Französisches Etymologisches Wörterbuch, Bonn-Leipzig-Berlin : F. Klopp.

GDLI = BATTAGLIA Salvatore, 1961-2000, Grande Dizionario della lingua italiana, Turin : UTET.

Huguet $=$ HuguET Edmond, 1928-1967, Dictionnaire de la langue française du seizième siècle, Paris : Honoré Champion.

Larousse des plantes = Mioulane Patrick, 2008, Le Grand Larousse des 15000 plantes et fleurs du jardin, Paris : Larousse.

Manuale di agricoltura $=$ HoEPLI Ulrico, 1997, Manuale di agricoltura. Climatologia, ambiente, agronomia, botanica, coltivazioni, ingegneria agraria, Milan : Hoepli.

TLFi = Version informatisée de IMBS Paul \& QuÉMADA Bernard, 1971-1994, Trésor de la langue française. Dictionnaire de la langue du XIX et du XX siècle, Paris : Editions du CNRS.

TLIO = Beltrami Pietro \& Leonardi Lino, 1997-2018, Tesoro della Lingua Italiana delle Origini, Rome : Opera del Vocabolario Italiano, CNR (Dictionnaire informatisé consultable en ligne).

\section{ANNEXE}

\section{Photos et reproductions}




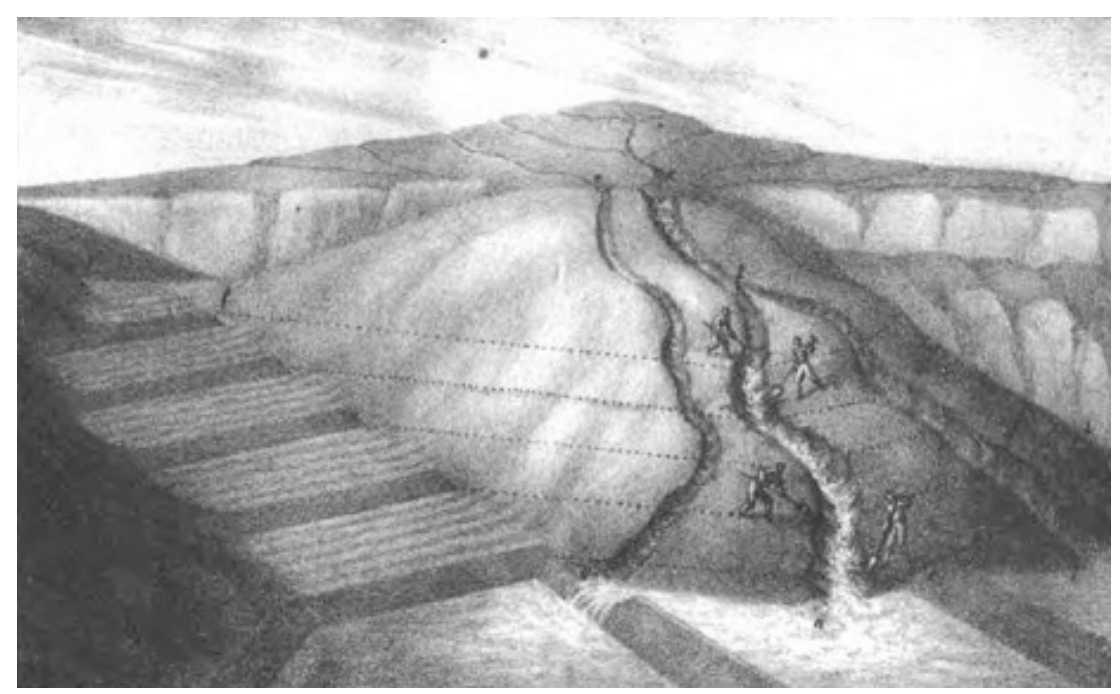

Fig. 1 Sistema della colmata toscana (reproduction de 1828)

Source : Sulle colmate di monte, Cosimo Ridolfi

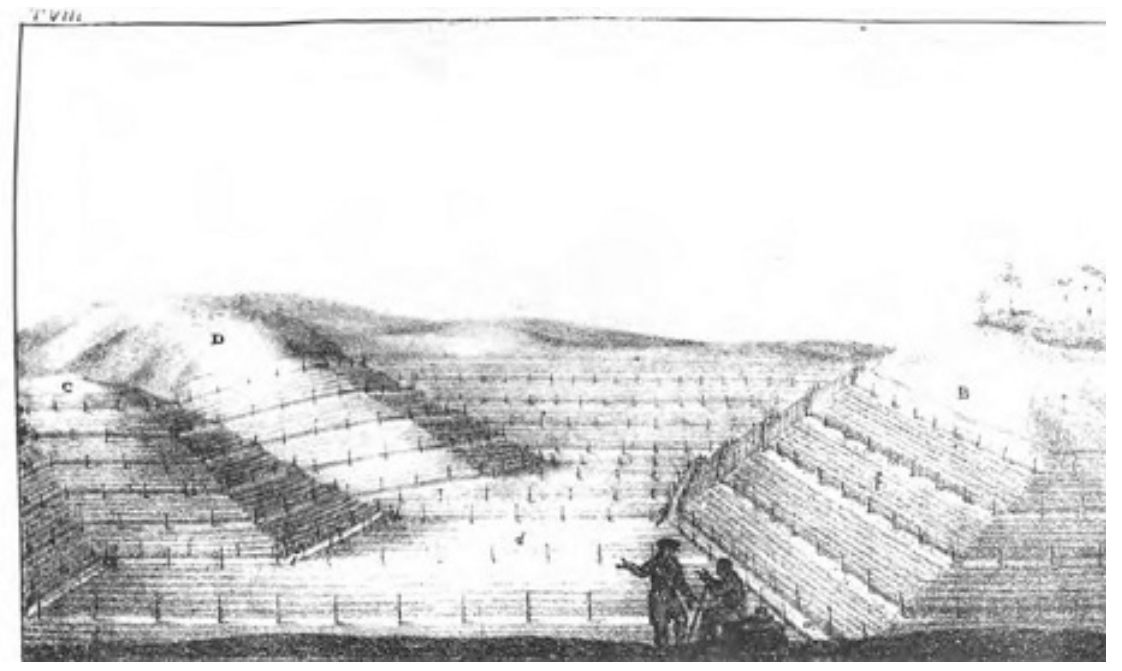

Fig. 2 Sistema della colmata toscana (reproduction de 1828) Sistema della "colmata" 